\title{
ON THE SMOOTHNESS OF CENTRES OF RATIONAL CHEREDNIK ALGEBRAS IN POSITIVE CHARACTERISTIC
}

\author{
GWYN BELLAMY \\ School of Mathematics and Statistics, University of Glasgow, \\ 15 University Gardens, Glasgow G12 8QW, United Kingdom \\ e-mail: gwyn.bellamy@glasgow.ac.uk \\ and MAURIZIO MARTINO \\ Mathematisches Institut, Endenicher Allee 60, 53115 Bonn, Germany \\ e-mail:mmartino@math.uni-bonn.de
}

Dedicated, with admiration and thanks, to Ken Brown and Toby Stafford on their 60th birthdays.

\begin{abstract}
In this paper we study rational Cherednik algebras at $t=1$ in positive characteristic. We study a finite-dimensional quotient of the rational Cherednik algebra called the restricted rational Cherednik algebra. When the corresponding pseudoreflection group belongs to the infinite series $G(m, d, n)$, we describe explicitly the block decomposition of the restricted algebra. We also classify all pseudo-reflection groups for which the centre of the corresponding rational Cherednik algebra is regular for generic values of the deformation parameter.
\end{abstract}

2010 Mathematics Subject Classification. 16G99, 16W70.

1. Introduction. Rational Cherednik algebras were introduced by Etingof and Ginzburg in 2002 [14]. Since their introduction they have been extensively studied and have been shown to be related to many other branches of mathematics such as integrable systems, symplectic algebraic geometry and algebraic combinatorics. In this paper we continue the study, initiated in $[\mathbf{1}, \mathbf{2}, \mathbf{6}, \mathbf{7}]$, of these algebras at $t=1$ and over a field of positive characteristic. We focus on the representation theoretical aspects of the story. In particular, we examine the block structure of certain finite-dimensional quotient algebras called restricted rational Cherednik algebras. We also look at the question of when the centre of the rational Cherednik algebra is smooth. These questions are motivated by the fact that the analogous problems for rational Cherednik algebras at $t=0$ in characteristic zero have important applications in symplectic algebraic geometry. Namely, it is know by the work of Ginzburg and Kaledin [16] that the centre of the rational Cherednik algebra associated to a group $W$ being smooth (at generic deformation parameter $\mathbf{c}$ ) is equivalent to the existence of a symplectic resolution for the quotient singularity $\left(V \times V^{*}\right) / W$. For each complex reflection group $W$, it is now known for which parameters $\mathbf{c}$, the centre of the corresponding rational Cherednik algebras at $t=0$ in characteristic zero, is smooth, see $[\mathbf{3}, \mathbf{4}, \mathbf{1 4}, \mathbf{1 7}, \mathbf{2 6}, \mathbf{2 8}]$. Our results are very similar in nature. The methods we develop, however, are new, and in fact can be used to reprove many of the characteristic zero results. 
1.1. Results. Let us review our results. Further details can be found in the main body of the paper. Let $W$ be a pseudo-reflection group. The main results of the paper are valid under the following restriction on the ground field:

$k$ is an algebraically closed field of characteristic $p>0$ with $p \nmid|W|$.

Let $V$ denote the reflection representation of $W$ over $k$. Let $\mathcal{S}(W)$ denote the set of reflections in $W$ and let $\mathbf{c}: \mathcal{S}(W) \rightarrow k$ be a $W$-invariant function. To this data we can attach a $k$-algebra $\mathrm{H}_{\mathbf{c}}(W)$ called the rational Cherednik algebra.

Let $V^{(1)}$ denote the Frobenius twist of $V$. The centre of $\mathrm{H}_{\mathbf{c}}(W)$ is denoted by $Z_{\mathbf{c}}(W)$. There is an injective algebra homomorphism

$$
k\left[V^{(1)}\right]^{W} \otimes k\left[\left(V^{*}\right)^{(1)}\right]^{W} \hookrightarrow Z_{\mathbf{c}}(W) .
$$

Factoring out by the unique graded, maximal ideal in this central subalgebra, one gets a finite-dimensional, graded quotient of the rational Cherednik algebra. This factor algebra is called the restricted rational Cherednik algebra and is denoted by $\overline{\mathrm{H}}_{\mathbf{c}}(W)$. Simple modules for this finite-dimensional algebra are in natural bijection with the simple modules of the group $W$. Thus, the blocks of $\overline{\mathrm{H}}_{\mathbf{c}}(W)$ give us a partition of the set Irr $W$. Our first main result is an explicit combinatorial description of this block partition when $W$ belongs to the infinite series $G(m, 1, n)$. In this case the irreducible representations of $W$ are naturally labelled by $\mathcal{P}(m, n)$, the set of $m$-multipartitions of $n$. For each $\lambda \in \mathcal{P}(m, n)$, let $L_{\mathbf{c}}(\lambda)$ denote the corresponding simple $\overline{\mathrm{H}}_{\mathbf{c}}(W)$-module. For the definitions in the following statement and the proof of the following theorem, see Section 4.4.

TheOREM. Let $\lambda, \boldsymbol{\mu} \in \mathcal{P}(m, n)$. Let $a=\left(0, H_{1}, H_{1}+H_{2}, \ldots, H_{1}+\cdots+H_{m-1}\right)$. Then $L_{\mathbf{c}}(\lambda)$ and $L_{\mathbf{c}}(\boldsymbol{\mu})$ belong to the same block of $\overline{\mathrm{H}}_{\mathbf{c}}(W)$ if and only if

$$
\sum_{i=0}^{m-1} x^{\left(a_{i}^{p}-a_{i}\right)} \operatorname{Res}_{\lambda^{i}}\left(x^{-\left(\kappa^{p}-\kappa\right)}\right)=\sum_{i=0}^{m-1} x^{\left(a_{i}^{p}-a_{i}\right)} \operatorname{Res}_{\mu^{i}}\left(x^{-\left(\kappa^{p}-\kappa\right)}\right) .
$$

Using this description of the blocks of the restricted rational Cherednik algebra $\overline{\mathrm{H}}_{\mathbf{c}}(G(m, 1, n))$, some Clifford theory also allows one to describe the blocks of the restricted rational Cherednik algebra $\overline{\mathrm{H}}_{\mathbf{c}}(G(m, d, n))$, see Section 4.5. Here $G(m, d, n)$ denotes (in the Shephard-Todd classification) the normal subgroup of $G(m, 1, n)$ where we impose the restriction that $d \mid m$ and either $n>2$ or $n=2$ and $d$ is odd.

1.2. Smoothness. Our remaining results concern the smoothness of $Z_{\mathbf{c}}(W)$. In Section 5 we relate the smoothness of $Z_{\mathbf{c}}(W)$ to the representation theory of $\mathrm{H}_{\mathbf{c}}(W)$. Taking as our starting point the fact that the smooth and Azumaya loci of $Z_{\mathbf{c}}(W)$ are equal, we use the restriction functors of [5] to establish the following.

THEOREM. The following are equivalent:

(1) $Z_{\mathbf{c}}(W)$ is smooth;

(2) the blocks of $\overline{\mathrm{H}}_{\mathbf{c}^{\prime}}\left(W^{\prime}\right)$ are singletons for all parabolic subgroups $W^{\prime} \subseteq W$.

Here $\mathbf{c}^{\prime}$ denotes the restriction of $\mathbf{c}$ to $\mathcal{S}(W) \cap W^{\prime}$. 
In the case that $W=G(m, 1, n)$, we can apply the theorem together with the description of the blocks from Theorem 1.1 to determine for which parameters $\mathbf{c}$ the centre $Z_{\mathbf{c}}(W)$ is smooth.

COROLlaRY. The centre of $\mathrm{H}_{\mathbf{c}}(G(m, 1, n))$ is smooth if and only if $\mathbf{c}$ does not lie on the finitely many hyperplanes in $\mathcal{C}$ defined by

$$
\kappa \in \mathbb{F}_{p} \quad \text { and } \quad a_{i}-a_{j} \pm C \kappa \in \mathbb{F}_{p}, \quad \forall 0 \leq i \neq j \leq m-1,0 \leq C \leq n-1 .
$$

We should note that an analogous version of the theorem and its corollary is true for rational Cherednik algebras at $t=0$ over the complex numbers. Using [28, Theorem 5.5], this gives an alternative way to describe the parameter values where the centre is smooth (c.f. [18, Lemma 4.3]). The theorem also clarifies the relationship between restricted Cherednik algebras and the smoothness of the centres $Z_{\mathbf{c}}(W)$.

1.3. A classification. Since $k=\bar{k}$ and hence $|k|=\infty$, the corollary shows that the centre of $\mathrm{H}_{\mathbf{c}}(G(m, 1, n))$ is a smooth algebra for generic values of the parameter $\mathbf{c}$. One can ask, more generally, the following question: For which pseudo-reflection groups $W$ is $Z_{\mathbf{c}}(W)$ smooth for generic values of the parameter $\mathbf{c}$ ? Our final result answers this question.

THEOREM. The centre of the rational Cherednik algebra $\mathrm{H}_{\mathbf{c}}(W)$ is never smooth if $W$ is not isomorphic to $G(m, 1, n)$ or $G_{4}$. If $W$ is isomorphic to $G(m, 1, n)$ for some $m$ and $n$ or to the exceptional group $G_{4}$, then the centre of $\mathrm{H}_{\mathbf{c}}(W)$ is smooth for generic values of $\mathbf{c}$.

Our approach to proving Theorem 1.3 follows the same path as in [3]- we calculate the Poincare polynomial of the graded $\overline{\mathrm{H}}_{\mathbf{c}}(W)$-modules $L_{\mathbf{c}}(\lambda)$ under the assumption that the dimension of these modules is maximal, namely $\operatorname{dim}_{k} L_{\mathbf{c}}(\lambda)=p^{n}|W|$. This leads to a contradiction for many choices of $\lambda$. However, the naive argument of loc. cit. is not sufficient in our case to calculate this Poincaré polynomial. Instead, we show that $L_{\mathbf{c}}(\lambda)$ can be deformed (flatly) to a graded $\mathrm{H}_{0}(W)=\mathcal{D}(V) \rtimes W$-module. Using a result of Cartier's on $\mathcal{D}$-modules with zero $p$-curvature, we study the graded $W$-character of this module at $\mathbf{c}=0$.

REMARK. Theorem 1.3 and Corollary 1.1 provide a complete answer to [7, Question D] for rational Cherednik algebras.

1.4. Structure of the paper. The paper is structured as follows. In Section 2 we introduce notation and recall some facts about pseudo-reflection groups. In Section 3 we define rational Cherednik algebras and state their main properties. The Dunkl-Opdam operators are introduced in Section 4 and are used to prove Theorem 1.1. Section 5 is devoted to parabolic restriction and induction and their application in the proof of Theorem 1.2. Finally, in Section 6 we establish certain properties of $\mathcal{D}$-modules in characteristic $p$ and use these to prove Theorem 1.3.

2. Basics. Let $k$ be a field of characteristic $p>0$. Let $V$ be a finite-dimensional $k$-vector space and $W$ be a finite group acting linearly on $V$. An element $s \in W$ is called a pseudo-reflection if the fixed space of $s$ has co-dimension one. Let $\mathcal{S}(W)$ 
denote the set of all pseudo-reflections in $W$. Then $W$ is said to be a pseudo-reflection group if $W=\langle\mathcal{S}(W)\rangle$, a good reference on the theory of pseudo-reflection groups is [25].

If the characteristic $p$ of the field $k$ does not divide the order of $W$ then there are no transvections in $\mathcal{S}(W)$. One can check from the classification of pseudo-reflection groups, as recalled in [24], that this assumption implies that $W$ is the reduction $\bmod p$ of a complex reflection group. Moreover, as noted in [33, Section 7.1], our assumption implies that each pseudo-reflection $s \in \mathcal{S}(W)$ is diagonalizable and hence the nontrivial eigenvalue of $s$ automatically belongs to $k$.

2.1. Frobenius twists and group actions. Again, let $V$ be a finite-dimensional vector space over $k$ and let $W$ be a finite group acting linearly on $V$. The Frobenius morphism Fr $: k[V] \rightarrow k[V]$ is the ring homomorphism $f \mapsto f^{p}$. Denote by $k\left[V^{(1)}\right]$ the image of Fr but with twisted linear structure $z \star f=z^{p} f$ for all $f \in k\left[V^{(1)}\right]$ and $z \in k$. Then Fr : $k[V] \rightarrow k\left[V^{(1)}\right]$ is a $k$-linear isomorphism. Note that $k[V]$ is a finite, free $k\left[V^{(1)}\right]$-module of rank $(\operatorname{dim} V)^{p}$. It is easy to check that Fr is a $W$-equivariant ring homomorphism, so we have an isomorphism $\mathrm{Fr}^{W}: k[V]^{W} \rightarrow k\left[V^{(1)}\right]^{W}$. In particular, the $p$ th powers of a generating set for $k[V]^{W}$ form a generating set for $k\left[V^{(1)}\right]^{W}$.

2.2. Representations of pseudo-reflection groups. Let $W$ be a finite group and $K$ be a finite field extension of $\mathbb{Q}$ containing all the $|W|$ th roots of unity. Let $A$ be the localisation of the ring of integers of $K$ at the prime ideal generated by $p \in \mathbb{Z}$, and let $L$ be the residue field of $A$. Note that $L$ is a finite field of order a power of $p$. With this setup we can define a decomposition map on characters of irreducible representations as follows, see [15, Section 7]. Let $M$ be an irreducible $K W$-module. We can choose an $A$-lattice $M_{A}$ in $M$ so that the action of $W$ on $M_{A}$ has structure constants in $A$. Let $M_{L}$ denote the reduction of $M_{A}$ to $L$, which is naturally a $L W$-module. The decomposition map is the assignment $\chi \mapsto \chi_{L}$, where $\chi, \chi_{L}$ denote the characters of $M$ and $M_{L}$ respectively. By [10, Corollary 17.2] and Tits' deformation theorem [15, Theorem 7.4.6], we have the following.

THEOREM. If $p$ does not divide $|W|$ then both $K W$ and $L W$ are split algebras and the decomposition map defines a bijection between the irreducible characters of $K W$ and $L W$.

In particular, it follows that if $k$ is algebraically closed of characteristic $p$ not dividing $|W|$ then the irreducible characters of $k W$ are given by reducing the irreducible characters of $\mathbb{C} W$ to $k$.

2.3. p-coinvariant rings. Let $W$ be a finite group and let $K_{0}(W)$ denote the Grothendieck group of finite dimensional $W$-modules. The image of $\lambda \in k W$-mod in $K_{0}(W)$ is denoted by $[\lambda]$. Let $\operatorname{Irr}_{k} W$ be a complete set of isomorphism classes of simple $W$-modules. We denote by $\operatorname{Irr}_{\mathbb{Z}} W$ a complete set of isomorphism classes of graded, simple $W$-modules. For a graded $W$-module $M$, we write $\operatorname{ch}_{t, W}(M) \in K_{0}(W)\left[t, t^{-1}\right]$ for its graded character. The shift $M[i]$ of $M$ is the graded $W$-module such that $M[i]_{j}=M_{j-i}$.

Assume that $W$ acts on the $k$-vector space $V$. We endow the algebra $k[V]$ with its usual $\mathbb{N}$-grading. The coinvariant ring of $W$ is $k[V]^{\operatorname{co} W}:=k[V] /\left\langle k[V]_{+}^{W}\right\rangle$, and for each 
$\lambda \in \operatorname{Irr} W$, we denote by $f_{\lambda}(t)$ the corresponding fake polynomial, defined by

$$
\operatorname{ch}_{t, W}\left(k[V]^{\operatorname{co} W}\right)=\sum_{\lambda \in \operatorname{Irr} W} f_{\lambda}(t) \cdot[\lambda] .
$$

Definition. Let $k\left[V^{(1)}\right]_{+}^{W}$ denote the invariant polynomials with zero constant term. The $p$-coinvariant ring is defined to be the finite-dimensional graded algebra $k[V]^{p \operatorname{co} W}:=k[V] /\left\langle k\left[V^{(1)}\right]_{+}^{W}\right\rangle$.

Now assume, moreover, that $W$ is a pseudo-reflection group and that the characteristic of $k$ is coprime to $|W|$. Then the Chevalley-Shephard-Todd Theorem (in this generality see [33, Theorem 7.4.1]) says that $k[V]^{W}$ is a polynomial ring. Let $d_{1}, \ldots, d_{n}$ be the degrees of a set of fundamental, homogeneous, algebraically independent generators of $k[V]^{W}$.

LEMMA. There is an isomorphism of graded $W$-modules

$$
\begin{aligned}
k[V]^{p \operatorname{co} W} & \simeq\left(k[V] /\left\langle k[V]_{+}^{W}\right\rangle\right) \otimes\left(k[V]^{W} /\left\langle k\left[V^{(1)}\right]_{+}^{W}\right\rangle\right) \\
& \simeq\left(k[V] /\left\langle k\left[V^{(1)}\right]_{+}\right\rangle\right) \otimes\left(k\left[V^{(1)}\right] /\left\langle k\left[V^{(1)}\right]_{+}^{W}\right\rangle\right) .
\end{aligned}
$$

Proof. Consider the inclusions of algebras

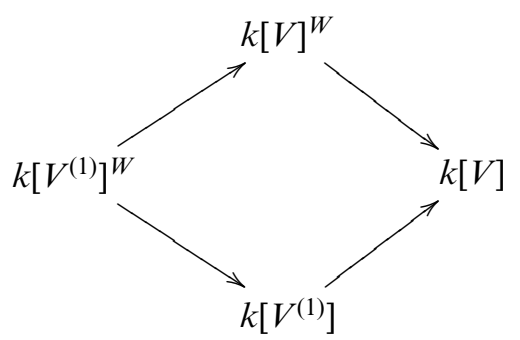

Choose a basis $x_{1}, \ldots, x_{n}$ of $V^{*}$, homogeneous generators $f_{1}, \ldots, f_{n}$ of $k[V]^{W}$ and a free homogeneous basis $b_{1}, \ldots, b_{|W|}$ of $k[V]$ as a $k[V]^{W}$-module. For $\alpha \in \mathbb{N}^{n}$, let $f^{\alpha}$, respectively $x^{\alpha}$, be the monomial $f_{1}^{\alpha_{1}} \cdots f_{n}^{\alpha_{n}}$, respectively $x_{1}^{\alpha_{1}} \cdots x_{n}^{\alpha_{n}}$. Then there are free bases for $k[V]$ as a $k\left[V^{(1)}\right]^{W}$-module given by

$$
\left\{f^{\alpha} b_{j}\left|0 \leq \alpha_{i} \leq p-1,1 \leq j \leq\right| W \mid\right\},
$$

and

$$
\left\{x^{\alpha} b_{j}^{p}\left|0 \leq \alpha_{i} \leq p-1,1 \leq j \leq\right| W \mid\right\} .
$$

Taking the images of these bases in $k[V]^{p c o W}$ yields the lemma.

REMARK. The isomorphism (1) implies that

$$
\sum_{i \in \mathbb{Z}}\left[k[V]_{i}^{p \operatorname{co} W}: \lambda\right] t^{i}=f_{\lambda}(t) \cdot \prod_{i=1}^{n} \frac{1-t^{p d_{i}}}{1-t^{d_{i}}}
$$

for all $\lambda \in \operatorname{Irr} W$. 
3. Rational Cherednik algebras. In this section, we define the rational Cherednik algebra and the restricted rational Cherednik algebra. Our presentation of $\mathrm{H}_{t, \mathbf{c}}(W)$ requires that $\mathcal{S}(W)$ contains no transvections. Therefore, we assume throughout this section that $p$, the characteristic of $k$, is coprime to $|W|$. There is a different presentation, given in [2], of the rational Cherednik algebra which is valid when $W$ contains transvection.

3.1. Definition. For $s \in \mathcal{S}(W)$, fix $\alpha_{s} \in V^{*}$ to be a basis of the one-dimensional space $\left.\operatorname{Im}(s-1)\right|_{V^{*}}$ and $\alpha_{s}^{\vee} \in V$ a basis of the one-dimensional space $\left.\operatorname{Im}(s-1)\right|_{V}$, normalised so that $\alpha_{s}\left(\alpha_{s}^{\vee}\right)=1$ (this is possible due to our assumption on $p$, see Section 2). Let $\mathcal{C}$ denote the space of $W$-equivariant functions $\mathcal{S}(W) \rightarrow k$ and choose $\mathbf{c} \in \mathcal{C}$ and $t \in k$. The rational Cherednik algebra, $\mathrm{H}_{t, \mathrm{c}}(W)$, as introduced by Etingof and Ginzburg $[14$, p. 250], is the quotient of the skew group algebra of the tensor algebra, $T(V \oplus$ $\left.V^{*}\right) \rtimes W$, by the ideal generated by the relations

$$
\left[x, x^{\prime}\right]=0, \quad\left[y, y^{\prime}\right]=0, \quad[y, x]=t x(y)-\sum_{s \in \mathcal{S}} \mathbf{c}(s) \alpha_{s}(y) x\left(\alpha_{s}^{\vee}\right) s,
$$

for all $x, x^{\prime} \in V^{*}$ and $y, y^{\prime} \in V$. We define a filtration $\mathcal{F}_{\bullet}$ on $\mathrm{H}_{t, \mathrm{c}}(W)$ via $\mathcal{F}_{0}=k W, \mathcal{F}_{1}=$ $k W \otimes\left(V \oplus V^{*}\right)$ and $\mathcal{F}_{i}=\mathcal{F}_{1}^{i}$ for $i>1$. By [14, Theorem 1.3], there is an isomorphism of algebras

$$
\operatorname{gr}_{\mathcal{F}} \mathrm{H}_{t, \mathbf{c}}(W) \cong S\left(V \oplus V^{*}\right) \rtimes W .
$$

As a consequence, there is a vector space isomorphism

$$
\mathrm{H}_{t, \mathrm{c}}(W) \cong k[V] \otimes k W \otimes k\left[V^{*}\right] .
$$

There is also a $\mathbb{Z}$-grading on $\mathrm{H}_{\mathbf{c}}(W)$ given by setting $\operatorname{deg}(W)=0, \operatorname{deg}(V)=-1$ and $\operatorname{deg}\left(V^{*}\right)=1$. Throughout this paper we assume that $t \neq 0$. Therefore, without loss of generality $t \equiv 1$ and we write $\mathrm{H}_{\mathrm{c}}(W)$ for $\mathrm{H}_{1, \mathrm{c}}(W)$. Let $x_{1}, \ldots, x_{n}$ be the basis of $V^{*}$ and $y_{1}, \ldots, y_{n} \in V$ the dual basis. Define the Euler element in $\mathrm{H}_{\mathbf{c}}(W)$ to be

$$
\mathbf{h}=\sum_{i=1}^{n} x_{i} y_{i}-\sum_{s \in S} \frac{c_{s}}{1-\lambda_{s}} S
$$

One can easily check that $[\mathbf{h}, x]=x,[\mathbf{h}, y]=-y$ and $[\mathbf{h}, w]=0$ for all $x \in V^{*}, y \in V$ and $w \in W$. Therefore, the element $\mathbf{h}^{p}-\mathbf{h}$ belongs to the centre of $\mathrm{H}_{\mathbf{c}}(W)$.

3.2. Basic properties of $\mathrm{H}_{t, \mathrm{c}}(W)$. Below we summarize the fundamental properties of $\mathrm{H}_{\mathrm{c}}(W)$. Proofs of all these statements can be found in [7]. Since some of these statements seem to require that $k$ be algebraically closed, we assume for the remainder of Section 3 that this is the case.

Proposition. Let $\mathrm{H}:=\mathrm{H}_{\mathbf{c}}(W)$ be a rational Cherednik algebra associated to $(V, W)$.

(1) The P.I. degree of $\mathrm{H}$ equals $p^{n}|W|$.

(2) The centre $Z_{\mathbf{c}}(W)$ of $\mathrm{H}$ is an affine domain and the algebra $\mathrm{H}$ is a finite module over its centre.

(3) The smooth locus of $Z_{\mathbf{c}}(W)$ equals the Azumaya locus of $\mathrm{H}$.

(4) The commutative subalgebras $k\left[V^{(1)}\right]^{W}$ and $k\left[\left(V^{*}\right)^{(1)}\right]^{W}$ of $\mathrm{H}$ are central. 
3.3. The restricted rational Cherednik algebra. Let $L$ be a simple, graded $\mathrm{H}_{\mathbf{c}}(W)$ module. The centre of $\mathrm{H}_{\mathbf{c}}(W)$ acts as a scalar on $L$ and the grading on $L$ implies that $\left(k\left[V^{(1)}\right]^{W} \otimes k\left[\left(V^{*}\right)^{(1)}\right]^{W}\right)_{+}$annihilates $L$. Therefore, to study these simple, graded modules, it suffices to consider a certain graded, finite-dimensional quotient of $\mathrm{H}_{\mathbf{c}}(W)$.

DEFINITION. The restricted rational Cherednik algebra $\overline{\mathrm{H}}_{\mathbf{c}}(W)$ is the finite-dimensional quotient of $\mathrm{H}_{\mathbf{c}}(W)$ by the central ideal generated by $\left(k\left[V^{(1)}\right]^{W} \otimes k\left[\left(V^{*}\right)^{(1)}\right]^{W}\right)_{+}$.

The algebra $\overline{\mathrm{H}}_{\mathbf{c}}(W)$ is $\mathbb{Z}$-graded and has dimension $p^{2 n}|W|^{3}$. The PoincaréBirkhoff-Witt property (5) implies that

$$
\overline{\mathrm{H}}_{\mathbf{c}}(W) \cong k[V]^{p \operatorname{co} W} \otimes k W \otimes k\left[V^{*}\right]^{p \operatorname{co} W},
$$

as vector spaces. Since $k[V]^{p c o W}$ is a complete intersection, [13, Corollary 21.19] implies that it is Gorenstein and thus equipped with a non-degenerate bilinear form. Then the proof of [9, Theorem 3.6] shows that the algebra $\overline{\mathrm{H}}_{\mathbf{c}}(W)$ is symmetric.

Definition. Let $\lambda \in \operatorname{Irr}_{\mathbb{Z}}(W)$. The baby Verma module $\bar{\Delta}(\lambda)$ associated to $\lambda$ is the induced module

$$
\bar{\Delta}(\lambda)=\overline{\mathrm{H}}_{\mathbf{c}}(W) \otimes_{A} \lambda,
$$

where $A=k\left[V^{*}\right]^{\text {pco } W} \rtimes W$ and the natural action of $W$ on $\lambda$ extends to $A$ by making $V$ act by zero.

As was done in [17], we can apply the theory developed in [21] to the category $\overline{\mathrm{H}}_{\mathrm{c}}(W)$-grmod of finite-dimensional, graded, left $\overline{\mathrm{H}}_{\mathbf{c}}(W)$-modules. The forgetful functor $\overline{\mathrm{H}}_{\mathbf{c}}(W)$-grmod $\rightarrow \overline{\mathrm{H}}_{\mathbf{c}}(W)$-mod is denoted as $F$.

Proposition. Let $\lambda, \mu \in \operatorname{Irr}_{\mathbb{Z}} W$.

(1) The baby Verma module $\bar{\Delta}(\lambda)$ has a simple head $L(\lambda)$.

(2) $\bar{\Delta}(\lambda)$ is isomorphic to $\bar{\Delta}(\mu)$ if and only if $\lambda=\mu$ in $\operatorname{Irr}_{\mathbb{Z}} W$.

(3) The set $\left\{L(\lambda) \mid \lambda \in \operatorname{Irr}_{\mathbb{Z}} W\right\}$ is a complete set of isomorphism classes of simple modules in $\overline{\mathrm{H}}_{\mathbf{c}}(W)$-grmod.

(4) The set $\left\{F(L(\lambda)) \mid \lambda \in \operatorname{Irr}_{\mathbb{Z}} W\right\}$ is a complete set of isomorphism classes of simple $\overline{\mathrm{H}}_{\mathbf{c}}(W)$-modules.

Proposition 3.3 shows that there is a natural bijection, $\lambda \mapsto L(\lambda)$, between $\operatorname{Irr}_{k} W$ and $\operatorname{Ir} \overline{\mathrm{H}}_{\mathbf{c}}(W)$. Therefore, the blocks of $\overline{\mathrm{H}}_{\mathbf{c}}(W)$ define a partition, which we call the block partition, of the set $\operatorname{Irr}_{k} W$. By Theorem 2.2, our assumption that $k$ is algebraically closed and $p$ coprime to $|W|$ implies that we can (and will) identify $\operatorname{Irr}_{k} W$ with $\operatorname{Irr}_{\mathbb{C}} W$.

4. Blocks for $G(m, d, n)$. In this section we carry out calculations (in a similar spirit of [27]) that lead to a proof of Theorem 1.1. We begin by fixing notation. Let $m \geq 1, n>1$ be integers. Let $C_{m}$ be the cyclic group of order $m$. We fix a generator $g \in C_{m}$ and let $s_{i j} \in S_{n}$ denote the transposition which swaps $i$ and $j$. We denote by $s_{j}$ the simple transposition swapping $j$ and $j+1$. The group $W=G(m, 1, n)$ is the semi-direct product $S_{n} \ltimes\left(C_{m}\right)^{n}$. We write $g_{i}^{l}$ for the element $\left(1, \ldots, g^{l}, \ldots 1\right) \in G(m, 1, n)$ with $g^{l}$ in the $i$ th place. Let $V=k^{n}$ be the reflection representation of $G(m, 1, n)$. Recall that we assume that $p \nmid|W|$, so in particular $p \neq 2$. Let $\eta \in k$ be a primitive $m$ th root of unity. 
We fix a basis $\left\{y_{1}, \ldots, y_{n}\right\}$ of $V$ so that

$$
g_{i}\left(y_{j}\right)=\left\{\begin{array}{l}
\eta y_{j} \text { if } i=j \\
y_{j} \text { otherwise }
\end{array} \quad \text { and } \sigma\left(y_{j}\right)=y_{\sigma(j)}\right.
$$

for all $i, j$ and all $\sigma \in S_{n}$. Let $\left\{x_{1}, \ldots, x_{n}\right\} \in V^{*}$ be the dual basis. The conjugacy classes of reflections in $W$ are given by

$$
\left\{s_{i j} g_{i}^{-l} g_{j}^{l}: 0 \leq l \leq m-1 \text { and } i \neq j\right\},
$$

and, for each $1 \leq l \leq m-1$,

$$
\left\{g_{j}^{l}: 1 \leq j \leq n\right\}
$$

The parameter $\mathbf{c}$ is represented by $\left(\kappa, c_{1}, \ldots, c_{m-1}\right) \in k^{n}$, where $\mathbf{c}\left(s_{i j} g_{i}^{-l} g_{j}^{l}\right)=\kappa$ and $\mathbf{c}\left(g_{j}^{l}\right)=c_{l}$. Using this notation, the definition of the rational Cherednik algebra becomes the following.

Definition. Let $W=G(m, 1, n)$. Then $\mathrm{H}_{\mathbf{c}}(W)$ is the quotient of $T\left(V \oplus V^{*}\right) \rtimes W$ by the relations:

$$
\begin{aligned}
& {\left[x_{i}, x_{j}\right]=0, \quad\left[y_{i}, y_{j}\right]=0,} \\
& {\left[y_{i}, x_{i}\right]=1-\kappa \sum_{l=0}^{m-1} \sum_{j \neq i} s_{i j} g_{i}^{-l} g_{j}^{l}-\sum_{l=1}^{m-1} c_{l}\left(1-\eta^{-l}\right) g_{i}^{l},} \\
& {\left[y_{i}, x_{j}\right]=\kappa \sum_{l=0}^{m-1} \eta^{-l} s_{i j} g_{i}^{-l} g_{j}^{l} .}
\end{aligned}
$$

4.1. The Dunkl-Opdam operators. Let $W=G(m, 1, n)$. A crucial feature in our arguments is the use of Dunkl-Opdam operators. These are the analogues of Jucys-Murphy elements for symmetric groups and (double) affine Hecke algebras. Applications to rational Cherednik algebras of type $G(m, d, n)$ were first considered by Dunkl and Opdam [12] and then extensively explored by Griffeth (and Dunkl) $[11,19,20]$. We begin by defining these elements and in the subsequent section calculating various formulae involving them.

For all $1 \leq i \leq n$, we define elements in $\mathrm{H}_{\mathbf{c}}(W)$ :

$$
\begin{aligned}
z_{i} & =y_{i} x_{i}-\frac{1}{2}+\kappa \sum_{l=0}^{m-1} \sum_{1 \leq j<i} s_{i j} g_{i}^{l} g_{j}^{-l}-\sum_{l=1}^{m-1} c_{l} \eta^{-l} g_{i}^{l} \\
& =x_{i} y_{i}+\frac{1}{2}-\kappa \sum_{l=0}^{m-1} \sum_{i<j} s_{i j} g_{i}^{l} g_{j}^{-l}-\sum_{l=1}^{m-1} c_{l} g_{i}^{l} .
\end{aligned}
$$

REMARK. The $z_{i}$ 's defined above are not quite the operators defined by Dunkl and Opdam [12], but the difference is minor. For more information, see [27, Remark 3.2].

In is an important fact proved by Griffeth [20] that $\left[z_{i}, z_{j}\right]=0$ for all $i, j$ (see also [27, Lemma 3.2]). Let $k\left[z_{1}, \ldots, z_{n}\right]$ denote the algebra generated by $z_{i}$ 's. By (4), this is a 
polynomial algebra. We denote by $E_{r}$ and $P_{r}$ the $r$ th elementary symmetric polynomial and $r$ th power sum in the $z_{i}$ respectively. By convention, $E_{0}=P_{0}=1$. We will use the following result from $[27,4.4]$, whose proof goes through verbatim over the field $k$.

THEOREM. We have

$$
\left[E_{r}, x_{1}\right]=\sum_{1<j_{2}<\cdots<j_{r} \leq n} x_{1} z_{j_{2}} \ldots z_{j_{r}}
$$

4.2. Computations. We now locate a useful subalgebra of the centre of the rational Cherednik algebra. We first give some simple combinatorial formulae comparing differentiation of elementary symmetric functions with that of elementary (Newton) power sums. We continue with the notation from Section 4.1. Let $E_{r}^{\prime}=\frac{d}{d z_{1}}\left(E_{r}\right)$ and $P_{r}^{\prime}=\frac{d}{d z_{1}}\left(P_{r}\right)=r z_{1}^{r-1}$. In this notation Theorem 4.1 reads

$$
\left[E_{r}, x_{1}\right]=x_{1} E_{r}^{\prime}
$$

Recall Newton's formula:

$$
r E_{r}=\sum_{i=1}^{r}(-1)^{i-1} P_{i} E_{r-i}
$$

Applying $\frac{d}{d z_{1}}$ to (11), we get

$$
r E_{r}^{\prime}=\sum_{i=1}^{r}(-1)^{i-1}\left(P_{i}^{\prime} E_{r-i}+P_{i} E_{r-i}^{\prime}\right) .
$$

A straightforward induction argument using the fact that $E_{r}^{\prime}=E_{r-1}-z_{1} E_{r-1}^{\prime}$ shows that

$$
E_{r}^{\prime}=\sum_{i=0}^{r-1}(-1)^{r-i-1} z_{1}^{r-i-1} E_{i}
$$

We will need a simple preparatory lemma. Let $Q_{n}:=\left(z_{1}+1\right)^{n}-z_{1}^{n}$.

LEMMA. We have

$$
Q_{n+1}=\sum_{i=1}^{n} z_{1}^{n-i} Q_{i}+(n+1) z_{1}^{n} .
$$

Proof. The proof is by induction. The case $n=0$ is clear. For $n>0$, we have

$$
Q_{n+1}=z_{1} Q_{n}+Q_{n}+z_{1}^{n},
$$

and the induction step follows by a simple calculation which we leave to the reader.

Proposition. For $1 \leq r \leq n$,

$$
\left[P_{r}, x_{1}\right]=x_{1} Q_{r}
$$


Proof. The proof goes by induction on $r$. For $r=1$ we have $P_{1}=E_{1}$ and $E_{1}^{\prime}=1$. By Theorem 4.1, $\left[P_{1}, x_{1}\right]=x_{1}$.

Suppose $r>1$. Then

$$
\begin{aligned}
(-1)^{r-1}\left[P_{r}, x_{1}\right] & =\left[r E_{r}+\sum_{i=1}^{r-1}(-1)^{i} P_{i} E_{r-i}, x_{1}\right] \\
& =r x_{1} E_{r}^{\prime}+\sum_{i=1}^{r-1}(-1)^{i}\left(P_{i} x_{1} E_{r-i}^{\prime}+x_{1} Q_{i} E_{r-i}\right) .
\end{aligned}
$$

The first line follows from rewriting $P_{r}$ using (11), and the second line follows from the induction hypothesis and (10). Now (14) equals

$$
\begin{aligned}
& r x_{1} E_{r}^{\prime}+\sum_{i=1}^{r-1}(-1)^{i}\left(x_{1} P_{i} E_{r-i}^{\prime}+x_{1} Q_{i}\left(E_{r-i}^{\prime}+E_{r-i}\right)\right) \\
= & x_{1}\left[\sum_{i=1}^{r}(-1)^{i-1}\left(P_{i}^{\prime} E_{r-i}+P_{i} E_{r-i}^{\prime}\right)+\sum_{i=1}^{r-1}(-1)^{i}\left(P_{i} E_{r-i}^{\prime}+Q_{i}\left(E_{r-i}^{\prime}+E_{r-i}\right)\right)\right] \\
= & x_{1}\left[\sum_{i=1}^{r}(-1)^{i-1} P_{i}^{\prime} E_{r-i}+\sum_{i=1}^{r-1}(-1)^{i}\left(Q_{i}\left(E_{r-i}^{\prime}+E_{r-i}\right)\right]\right. \\
= & x_{1}\left[\sum_{i=1}^{r}(-1)^{i-1} i z_{1}^{i-1} E_{r-i}+\sum_{i=1}^{r-1}(-1)^{i}\left(Q_{i}\left(\sum_{k=0}^{r-i-1}(-1)^{r-i-k-1} z_{1}^{r-i-k-1} E_{k}\right)+Q_{i} E_{r-i}\right)\right] .
\end{aligned}
$$

Here the first line follows from the induction hypothesis, the second from (12) and the fourth from (13). For a fixed $1 \leq l \leq r-1$, the coefficient of $E_{r-l}$ in (15) is equal to

$$
(-1)^{l-1} l z_{1}^{l-1}+\sum_{i=1}^{l-1}(-1)^{l-1} z_{1}^{l-i-1} Q_{i}+(-1)^{l} Q_{l},
$$

which equals zero by the above lemma. By the above lemma, the coefficient of $E_{0}$ is

$$
(-1)^{r-1} r z^{r-1}+\sum_{i=1}^{r-1}(-1)^{r-1} z_{1}^{r-i-1} Q_{i}=(-1)^{r-1} Q_{r}
$$

Therefore, we have $\left[P_{r}, x_{1}\right]=x_{1} Q_{r}$ as required.

4.3. Symmetric Dunkl-Opdam operators. We can now provide some central elements in $\mathrm{H}_{\mathbf{c}}(W)$. Recall from [27, 3.3] that there is an algebra isomorphism $\psi: \mathrm{H}_{\mathbf{c}}(W) \rightarrow$ $\mathrm{H}_{\mathbf{c}^{\prime}}(W)$, where $\mathbf{c}^{\prime}=\left(\kappa^{\prime}, c_{1}^{\prime}, \ldots, c_{m-1}^{\prime}\right)$ is the parameter with $\kappa^{\prime}=-\kappa$ and $c_{l}^{\prime}=\eta^{l} c_{-l}$ for all $l$. The isomorphism $\psi$ is given by $\psi\left(x_{i}\right)=y_{n-i+1}, \psi\left(y_{i}\right)=x_{n-i+1}, \psi\left(s_{i}\right)=s_{n-i}$ and $\psi\left(g_{i}\right)=g_{n-i+1}^{-1}$ for all $i$. Thus, $\psi\left(z_{i}\right)=z_{n-i+1}$ for all $i$. 
THEOREM. For all $1 \leq r \leq n$,

$$
P_{r}\left(z_{1}^{p}-z_{1}, \ldots, z_{n}^{p}-z_{n}\right)=\sum_{i=1}^{n}\left(z_{i}^{p}-z_{i}\right)^{r} \in Z_{\mathbf{c}}(W) .
$$

Thus, $k\left[z_{1}^{p}-z_{1}, \ldots, z_{n}^{p}-z_{n}\right]^{S_{n}} \subset Z_{\mathbf{c}}(W)$.

Proof. We continue to write $P_{m}$ for the power sum in the variables $z_{1}, \ldots, z_{n}$. We have

$$
\sum_{i=1}^{n}\left(z_{i}^{p}-z_{i}\right)^{r}=\sum_{i=1}^{n} \sum_{j=0}^{r}\left(\begin{array}{l}
r \\
j
\end{array}\right)(-1)^{r-j} z_{i}^{p j} z_{i}^{r-j}=\sum_{j=0}^{r}\left(\begin{array}{l}
r \\
j
\end{array}\right)(-1)^{r-j} P_{p j+r-j}
$$

Therefore by Proposition 4.2,

$$
\begin{aligned}
{\left[\sum_{i=1}^{n}\left(z_{i}^{p}-z_{i}\right)^{r}, x_{1}\right] } & =x_{1} \sum_{j=0}^{r}\left(\begin{array}{l}
r \\
j
\end{array}\right)(-1)^{r-j} Q_{p j+r-j} \\
& =x_{1} \sum_{j=0}^{r}\left(\begin{array}{l}
r \\
j
\end{array}\right)(-1)^{r-j}\left(\left(z_{1}+1\right)^{p j+r-j}-z_{1}^{p j+r-j}\right) \\
& =x_{1} \sum_{j=0}^{r}\left(\begin{array}{l}
r \\
j
\end{array}\right)(-1)^{r-j}\left(\left(z_{1}^{p}+1\right)^{j}\left(z_{1}+1\right)^{r-j}-\left(z_{1}^{p}\right)^{j} z_{1}^{r-j}\right) \\
& =x_{1}\left[\left(z_{1}^{p}+1\right)-\left(z_{1}+1\right)\right]^{r}-x_{1}\left(z_{1}^{p}-z_{1}\right)^{r}=0 .
\end{aligned}
$$

The theorem now follows as in [27, Theorem 3.4]. The element $P_{r}\left(z_{1}^{p}-z_{1}, \ldots, z_{n}^{p}-z_{n}\right)$ is symmetric in $z_{i}$ 's so it commutes with any $\sigma \in S_{n}$, see [20, Lemma 5.1]. Therefore, this power sum commutes with $\sigma x_{1} \sigma^{-1}=\sigma\left(x_{1}\right)$ for all $\sigma$, and in particular with each $x_{i}$. To see that the power sum also commutes with $y_{i}$ 's, we use the isomorphism $\psi$ from the paragraph above. Applying $\psi^{-1}$ to $\left[P_{r}\left(z_{1}^{p}-z_{1}, \ldots, z_{n}^{p}-z_{n}\right), x_{i}\right]$ shows that $\left[P_{r}\left(z_{1}^{p}-z_{1}, \ldots, z_{n}^{p}-z_{n}\right), y_{i}\right]=0$ for all $i$.

4.4. Blocks. We use an identical argument to the proof of [27, Theorem 5.5] to determine the blocks of $\overline{\mathrm{H}}_{c}(W)$. Let us first change our parameters. We define $h, H_{0}, \ldots, H_{m-1} \in k$ via

$$
h=-\kappa \quad \text { and } \quad-c_{l}\left(1-\eta^{-l}\right)=\sum_{j=0}^{m-1} \eta^{-l j} H_{j} .
$$

We denote by $\mathcal{P}(m, n)$ the set of $m$-multipartitions of $n, \mathcal{P}(m, n):=\left\{\left(\lambda^{0}, \ldots, \lambda^{m-1}\right)\right.$ : $\left.\sum_{i=0}^{m-1}\left|\lambda^{i}\right|=n\right\}$. The simple representations of $k W$ are labelled by the set $\mathcal{P}(m, n)$; over the complex numbers this is standard (see [22] or [32]) and the same holds for $k W$ by reduction (cf. Section 2.2). In fact, the construction from [32] is also valid over $k$, since $p$ does not divide $|W|$ so that we can find bases of irreducible $k W$-modules given by eigenvectors of Jucys-Murphy elements for the group $W$ (cf. [27, Section 5.3].

We recall the notion of the (shifted) residue of a multipartition, for more details see [27, Section 5.2]. Let $\lambda$ be a partition, which we identify with its Young diagram. The content of a box $b$ in $\lambda$ is the number $\operatorname{ct}(b):=j-i$, where 
$b$ lies in column $j$ and row $i$ of $\lambda$. The residue of a partition $\lambda$ is the polynomial $\operatorname{Res}_{\lambda}(x):=\sum_{b \in \lambda} x^{\operatorname{ct}(b)}$. For $\lambda=\left(\lambda^{0}, \ldots, \lambda^{m-1}\right) \in \mathcal{P}(m, n)$ and $\mathbf{a}=\left(a_{1}, \ldots, a_{m}\right) \in k^{m}$, let $\operatorname{Res}^{\mathrm{a}} \lambda:=\sum_{i=0}^{m-1} x^{\left(a_{i}^{p}-a_{i}\right)} \operatorname{Res}_{\lambda^{i}}\left(x^{-\left(\kappa^{p}-\kappa\right)}\right)$.

THEOREM. Let $\boldsymbol{\lambda}, \boldsymbol{\mu} \in \mathcal{P}(m, n)$. Let $\mathbf{a}=\left(0, H_{1}, H_{1}+H_{2}, \ldots, H_{1}+\cdots+H_{m-1}\right)$. Then $L_{\mathfrak{c}}(\lambda)$ and $L_{\mathfrak{c}}(\mu)$ belong to the same block of $\overline{\mathrm{H}}_{\mathrm{c}}(W)$ if and only if

$$
\operatorname{Res}^{\mathrm{a}} \lambda=\operatorname{Res}^{\mathrm{a}} \boldsymbol{\mu} .
$$

Proof. Since this follows the proof of [27, Theorem 5.5] closely, we shall sketch the argument, pointing out the necessary changes to loc. cit. Using Theorem 4.3 together with Weyl's Theorem for invariants of symmetric groups (which is valid whenever $p \nmid|W|$, see [33, Theorem 3.3.1]), it is enough to calculate the characters of $P_{r}\left(z_{1}^{p}-z_{1}, \ldots, z_{n}^{p}-z_{n}\right)$ on each $L_{\mathrm{c}}(\lambda)$. The characters are determined by choosing a simultaneous eigenvector $v_{\lambda} \in L_{\mathfrak{c}}(\lambda)$ for $z_{1}, \ldots, z_{n}$. The eigenvalues for $z_{i}^{p}-z_{i}$ are evaluated as in [27, Section 5.4], and produce the desired combinatorial description.

4.5. Blocks for $G(m, d, n)$. The blocks of $\overline{\mathrm{H}}_{c}(W)$ for $W=G(m, d, n)$, where $d \mid m$ and either $n>2$ or $n=2$ and $d$ is odd, can be calculated from Theorem 4.4 by using Clifford theory as in [4]. The resulting description of the blocks is then analogous to that given in characteristic zero, see [27, Section 5.6].

5. Smoothness of centres for $G(m, 1, n)$. In this section we assume that $k$ is a field of characteristic $p$ and $W$ is an arbitrary pseudo-reflection group such that $p$ is prime to the order of $|W|$. The main result of this section, Corollary 5.5, shows that the centre $Z_{\mathbf{c}}(W, V)$ of the rational Cherednik algebra being a regular algebra is equivalent to the block partition of $\overline{\mathrm{H}}_{\mathrm{c}^{\prime}}\left(W^{\prime}\right)$ consisting of singletons for all parabolic subgroups $W^{\prime}$ of $W$. Our proof of this result is based on identifying, as in [5], a certain completion of $\mathrm{H}_{\mathrm{c}}(W)$ with an algebra of matricies over the completion of $\mathrm{H}_{\mathrm{c}^{\prime}}\left(W^{\prime}\right)$ for a suitable parabolic subgroup $W^{\prime}$ of $W$. Applying Corollary 5.5 to the results of the previous section, we describe precisely for which parameters $\mathbf{c}$ the algebra $Z_{\mathbf{c}}(W, V)$ is regular when $W=G(m, 1, n)$.

5.1. Dunkl embedding. Let $\alpha=\prod_{s \in S} \alpha_{s}$. Let $V_{\text {reg }}$ denote the set $\{v \in V \mid \alpha(v) \neq 0\}$. Let $\mathcal{D}\left(V_{\text {reg }}\right)$ be the algebra of crystalline differential operators on $V_{\text {reg. }}$. For $y \in V$, the Dunkl operator associated to $y$ is

$$
D_{y}=\partial_{y}+\sum_{s \in \mathcal{S}} \frac{\mathbf{c}(s)}{1-\lambda_{s}} \frac{\alpha_{s}(y)}{\alpha_{s}}(s-1) \in \mathcal{D}\left(V_{\mathrm{reg}}\right) \rtimes W .
$$

By $[\mathbf{1 4}, \mathrm{p} .280]$ there is an injective algebra morphism

$$
\mathrm{H}_{\mathbf{c}}(W) \hookrightarrow \mathcal{D}\left(V_{\mathrm{reg}}\right) \rtimes W ; \quad w \mapsto w, x \mapsto x, y \mapsto D_{y}
$$

for all $w \in W, x \in V^{*}$ and $y \in V$. This embedding becomes an isomorphism after localizing $\mathrm{H}_{\mathbf{c}}(W)$ at the Ore set $\left\{\alpha^{m}\right\}_{m \geq 0}$. 
5.2. Completions. Let us recall the setup of [5]. Let $W^{\prime} \subset W$ be the stabilizer of a point $b \in V$ and let $\bar{V}=V / V^{W^{\prime}}$. For any closed $K$-point $b \in V$ (where $k \subset K$ is a finite field extension), we write $k[[V]]_{b}$ for the completion of $k[V]$ at $b$, and we write $\left.\widehat{k[V}\right]_{b}$ for the completion of $k[V]$ at the $W$-orbit of $b$ in $V$. Note that we have $k[[V]]_{0}=k[V]_{0}$. For any finitely generated $k[V]$-module $M$, let

$$
\left.\widehat{M}_{b}=\widehat{k[V}\right]_{b} \otimes_{k[V]} M .
$$

The completion $\widehat{\mathrm{H}}_{\mathbf{c}}(W, V)_{b}$ is the algebra generated by $\left.k \widehat{[V}\right]_{b}$, the Dunkl operators $D_{y}$ for $y \in V$ and the group $W$. Let $\mathbf{c}^{\prime}$ denote the restriction of $\mathbf{c}$ to $S \cap W^{\prime}$. The algebra $\widehat{\mathrm{H}}_{\mathbf{c}^{\prime}}\left(W^{\prime}, V\right)_{0}$ is then defined similarly. Let $P=\operatorname{Fun}_{W^{\prime}}\left(W, \widehat{\mathrm{H}}_{\mathbf{c}}\left(W^{\prime}, V\right)_{0}\right)$ be the set of $W^{\prime}$-equivariant maps from $W$ to $\widehat{\mathrm{H}}_{\mathbf{c}}\left(W^{\prime}, V\right)_{0}$. Let $Z\left(W, W^{\prime}, \widehat{\mathrm{H}}_{\mathbf{c}}\left(W^{\prime}, V\right)_{0}\right)$ be the ring of endomorphisms of the right $\widehat{\mathrm{H}}_{\mathbf{c}}\left(W^{\prime}, V\right)_{0}$-module $P$. The following proposition is proved over $\mathbb{C}$ in [5, Theorem 3.2], and has an identical proof over $k$.

Proposition. There is an isomorphism of algebras

$$
\Theta: \widehat{\mathrm{H}}_{\mathbf{c}}(W, V)_{b} \rightarrow Z\left(W, W^{\prime}, \widehat{\mathrm{H}}_{\mathbf{c}^{\prime}}\left(W^{\prime}, V\right)_{0}\right)
$$

defined as follows: for $f \in P, \alpha \in V^{*}, a \in V, u \in W$,

$$
\begin{aligned}
(\Theta(u) f)(w) & =f(w u), \\
\left(\Theta\left(x_{\alpha}\right) f\right)(w) & =\left(x_{w \alpha}^{(b)}+\alpha\left(w^{-1} b\right)\right) f(w), \\
\left(\Theta\left(y_{a}\right) f\right)(w) & =y_{w a}^{(b)} f(w)+\sum_{s \in \mathcal{S}, s \notin W^{\prime}} \frac{\mathbf{c}(s)}{1-\lambda_{s}} \frac{\alpha_{s}(w a)}{x_{\alpha_{s}}^{(b)}+\alpha_{s}(b)}(f(s w)-f(w)),
\end{aligned}
$$

where $x_{\alpha} \in V^{*} \subset \mathrm{H}_{\mathbf{c}}(W, V), x_{\alpha}^{(b)} \in V^{*} \subset \mathrm{H}_{\mathbf{c}^{\prime}}\left(W^{\prime}, V\right), y_{a} \in V \subset \mathrm{H}_{\mathbf{c}}(W, V)$ and $y_{a}^{(b)} \in$ $V \subset \mathrm{H}_{\mathbf{c}^{\prime}}\left(W^{\prime}, V\right)$.

Let $e_{11} \in Z\left(W, W^{\prime}, \widehat{\mathrm{H}}_{\mathbf{c}^{\prime}}\left(W^{\prime}, V\right)_{0}\right)$ be defined by $\left(e_{11} \cdot f\right)(w)=f(w)$ if $w \in W^{\prime}$ and $\left(e_{11} \cdot f\right)(w)=0$ otherwise. Then $e_{11}$ is a primitive idempotent and $\widehat{\mathrm{H}}_{\mathbf{c}^{\prime}}\left(W^{\prime}, V\right)_{0} \cong$ $e_{11} Z\left(W, W^{\prime}, \widehat{\mathrm{H}}_{\mathbf{c}^{\prime}}\left(W^{\prime}, V\right)_{0}\right) e_{11}$. The bimodule $e_{11} Z\left(W, W^{\prime}, \widehat{\mathrm{H}}_{\mathbf{c}^{\prime}}\left(W^{\prime}, V\right)_{0}\right)$ yields a Morita equivalence between $\widehat{\mathrm{H}}_{\mathbf{c}^{\prime}}\left(W^{\prime}, V\right)_{0}$ and $Z\left(W, W^{\prime}, \widehat{\mathrm{H}}_{\mathbf{c}^{\prime}}\left(W^{\prime}, V\right)_{0}\right)$.

5.3. Small representations. Suppose that $M$ is a finite-dimensional $\mathrm{H}_{\mathbf{c}}(W, V)$-module and let $\operatorname{Supp} M$ denote the support of $M$ as a $k[V]^{W}$-module. If $\operatorname{Supp} M$ is the $W$ orbit of some closed $K$-point $b \in V$, then $M$ is naturally a $\widehat{\mathrm{H}}_{c}(W, V)_{b}$-module. In particular, the support of any simple $\mathrm{H}_{\mathbf{c}}(W, V)$-module is a single $W$-orbit and so is a $\widehat{\mathrm{H}}_{\mathbf{c}}(W, V)_{b}$-module. Let $M$ be a $\widehat{\mathrm{H}}_{\mathbf{c}}(W, V)_{b}$-module, then denote by $\Theta^{*} M$ the $Z\left(W, W^{\prime}, \widehat{\mathrm{H}}_{\mathbf{c}^{\prime}}\left(W^{\prime}, V\right)_{0}\right)$-module given by transporting the $\widehat{\mathrm{H}}_{\mathbf{c}}(W, V)_{b}$-action on $M$ via $\Theta$.

Definition. An $\mathrm{H}_{\mathbf{c}}(W, V)$-module $M$ is called small if $\operatorname{dim}_{k} M<|W| p^{n}$.

Proposition. $A \mathrm{H}_{\mathbf{c}}(W, V)$-module $M$ is small if and only if $e_{11} \Theta^{*}(M)$ is a small $\mathrm{H}_{\mathbf{c}^{\prime}}\left(W^{\prime}, V\right)$-module.

Proof. Firstly, note that $e_{11} \Theta^{*}(M)$ is a $\mathrm{H}_{\mathbf{c}^{\prime}}\left(W^{\prime}, V\right)$-module by restricting the $\widehat{\mathrm{H}}_{\mathbf{c}^{\prime}}\left(W^{\prime}, V\right)_{0}$-action. Let $g_{1}=1, \ldots, g_{t}$ be right coset representatives of $W^{\prime}$ in $W$. Then $\Theta^{*}(M)=\bigoplus_{i=1}^{t} g_{i} e_{11} g_{i}^{-1} \Theta^{*} M$, with

$$
\operatorname{dim}_{k} g_{i} e_{11} g_{i}^{-1} \Theta^{*} M=\operatorname{dim}_{k} g_{j} e_{11} g_{j}^{-1} \Theta^{*} M, \quad \forall i, j .
$$


In particular, $\operatorname{dim} M<|W| p^{n}$ if and only if $\operatorname{dim} e_{11} \Theta^{*}(M)<|W| p^{n} / t=\left|W^{\prime}\right| p^{n}$.

5.4. Support. For the remainder of Section 5, we assume that $k$ is algebraically closed. Let $Z_{\mathbf{c}}(W, V)$ denote the centre of $\mathrm{H}_{\mathbf{c}}(W, V)$.

COROLlaRY. Keep notation as above. Then $Z_{\mathbf{c}}(W, V)$ is smooth if and only if $\mathrm{H}_{\mathbf{c}^{\prime}}\left(W^{\prime}, V\right)$ has no small modules $M$ with $\operatorname{Supp} M=0$ for all parabolic subgroups $W^{\prime} \subseteq W$.

Proof. By Proposition $3.2(3), Z_{\mathbf{c}}(W, V)$ is smooth if and only if $\mathrm{H}_{\mathbf{c}}(W, V)$ has no small modules. Let $M$ be a small, simple $\mathrm{H}_{\mathbf{c}}(W, V)$-module and choose $b \in V$ such that the support of $M$ equals the $W$-orbit of $b$. Then $M$ extends to a $\widehat{\mathrm{H}}_{\mathbf{c}}(W, V)_{b}$-module and Proposition 5.3 says that $e_{11} \Theta^{*}(M)$ is a small $\mathrm{H}_{\mathbf{c}^{\prime}}\left(W^{\prime}, V\right)$-module, where $W^{\prime}$ is the stabilizer of $b$ in $W$. Moreover, the support of $e_{11} \Theta^{*}(M)$ is 0 . Conversely, if there exists a small $\mathrm{H}_{\mathbf{c}^{\prime}}\left(W^{\prime}, V\right)$-module $M^{\prime}$ supported on 0 then this extends to a $\widehat{H}_{\mathbf{c}^{\prime}}\left(W^{\prime}, V\right)_{0}$-module. Since $\widehat{\mathrm{H}}_{\mathbf{c}^{\prime}}\left(W^{\prime}, V\right)_{0}$ is Morita equivalent to $Z\left(W, W^{\prime}, \widehat{\mathrm{H}}_{\mathbf{c}^{\prime}}\left(W^{\prime}, V\right)_{0}\right) \cong \widehat{\mathrm{H}}_{\mathbf{c}}(W, V)_{b}$, there exists some $\widehat{\mathrm{H}}_{\mathbf{c}}(W, V)_{b}$-module $M$ such that $M^{\prime} \simeq e_{11} \Theta^{*}(M)$. Proposition 5.3 says that $M$ is actually a small $\mathrm{H}_{\mathbf{c}}(W, V)$-module.

5.5. The proof of Theorem 1.3. Let $M$ be a simple $\mathrm{H}_{\mathbf{c}}(W, V)$-module with $\operatorname{Supp} M=0$. We can define an induced module (cf. 3.3) as follows. Let $B=k[V] \rtimes W$ and let $E \in \operatorname{Irr} W$. Extend the $W$-action on $E$ to a $B$-action by letting $f \in k[V]$ act by $f(0)$. Define

$$
\Delta(E)=\mathrm{H}_{\mathbf{c}}(W, V) \otimes_{B} E .
$$

By the support condition on $M$, the subspace $M_{0}=\left\{m \in M \mid V^{*} \cdot m=0\right\}$ of $M$ is non-zero. It is a $W$-submodule of $M$ and we may assume without loss of generality that $E \subseteq M_{0}$. Therefore, there is a surjective homomorphism $\Delta(E) \rightarrow M$, which maps $1 \otimes E \subset \Delta(E)$ to $E \subseteq M_{0}$ in the obvious way. Recall that $\mathrm{H}_{\mathbf{c}}(W, V)$ has a $\mathbb{Z}$-grading. We make $E$ into a graded $B$-module by setting $E_{0}=E$ and $E_{i}=0$ for all $i \neq 0$. We give $\Delta(E)$ a $\mathbb{Z}$-graded module structure by inducing the graded structure on $E$. Since $M$ is simple, there is some $a \in\left(V^{*}\right)^{(1)} / W$ such that $\mathfrak{m}_{a} \cdot M=0$, where $\mathfrak{m}_{a}$ denotes the maximal ideal corresponding to $a$. Define

$$
\Delta(a, E)=\left(\mathrm{H}_{\mathbf{c}}(W, V) \otimes_{B} E\right) / \mathfrak{m}_{a} \cdot\left(\mathrm{H}_{\mathbf{c}}(W, V) \otimes_{B} E\right) .
$$

By our choice of $a$, there is also a surjective homomorphism $\Delta(a, E) \rightarrow M$. The grading on $\Delta(E)$ induces filtrations on $\Delta(a, E)$ and $M$. We use the notation $\mathrm{gr}_{\mathbb{Z}}$ to denote the associated graded objects with respect to these filtrations.

Recall that a morphism $f: M \rightarrow N$ between filtered $\mathrm{H}_{\mathbf{c}}(W, V)$-modules is called a strictly filtered morphism if $f\left(F_{i} M\right)=F_{i} N \cap f(M)$ for all $i \in \mathbb{Z}$. The functor $\mathrm{gr}_{\mathbb{Z}}$ is exact on short exact sequences of strictly filtered morphisms. Also note that the surjection $\Delta(a, E) \rightarrow M$ is strictly filtered by definition. The filtration on $\Delta(E)$ is both exhaustive and separating, therefore the same is true of $\Delta(a, E)$. However, this module is finite-dimensional, therefore we have $F_{i} \Delta(a, E)=0$ and $F_{-i} \Delta(a, E)=\Delta(a, E)$ for $i \gg 0$.

Proposition. The $\mathrm{H}_{\mathbf{c}}(W, V)$-module $\operatorname{gr}_{\mathbb{Z}} M$ has dimension $\operatorname{dim}_{k} M$ and is annihilated by $\left(k\left[V^{(1)}\right]^{W} \otimes k\left[\left(V^{*}\right)^{(1)}\right]^{W}\right)_{+}$. 
Proof. Denote by $F_{i}$ the $i$ th piece of the filtration on $M$. By construction, $F_{\bullet}$ is a decreasing filtration with $F_{1}=0$ and $F_{-i}=M$ for all $i \gg 0$. Thus, $\operatorname{dim}_{k} \operatorname{gr}_{\mathbb{Z}} M=$ $\sum_{i=0}^{\infty} \operatorname{dim}_{k} F_{-i} / F_{-i+1}=\operatorname{dim}_{k} M$.

For the second statement, we show that $\operatorname{gr}_{\mathbb{Z}} \Delta(a, E) \cong \Delta(0, E)$. The claim then follows since there is a surjective map $\Delta(0, E) \rightarrow \operatorname{gr}_{\mathbb{Z}} M$. Consider the short exact sequence:

$$
0 \rightarrow \mathfrak{m}_{a} \cdot\left(\mathrm{H}_{\mathbf{c}}(W, V) \otimes_{B} E\right) \rightarrow \Delta(E) \rightarrow \Delta(a, E) \rightarrow 0,
$$

where the left-hand term is given the induced filtration. By the PBW theorem and the Nullstellensatz, $\operatorname{gr}_{\mathbb{Z}} \mathfrak{m}_{a} \cdot\left(\mathrm{H}_{\mathbf{c}}(W, V) \otimes_{B} E\right)=k\left[\left(V^{*}\right)^{(1)}\right]_{+}^{W} \cdot\left(\mathrm{H}_{\mathbf{c}}(W, V) \otimes_{B} E\right)$. Since $\Delta(E)$ is graded, $\operatorname{gr}_{\mathbb{Z}} \Delta(E)=\Delta(E)$. Thus, $\operatorname{gr}_{\mathbb{Z}} \Delta(a, E) \cong \Delta(0, E)$.

COROLLARY. The following are equivalent:

(1) $Z_{\mathbf{c}}(W, V)$ is smooth;

(2) $\mathrm{H}_{\mathbf{c}^{\prime}}\left(W^{\prime}, \bar{V}\right)$ has no small modules $M$ with $\operatorname{Supp} M=0$ for all parabolic subgroups $W^{\prime} \subseteq W$

(3) $\overline{\mathrm{H}}_{\mathbf{c}^{\prime}}\left(W^{\prime}, \bar{V}\right)$ has no small modules $M$ for all parabolic subgroups $W^{\prime} \subseteq W$;

(4) the blocks of $\overline{\mathrm{H}}_{\mathrm{c}^{\prime}}\left(W^{\prime}, \bar{V}\right)$ are singletons for all parabolic subgroups $W^{\prime} \subseteq W$.

Proof. There are isomorphisms of algebras $\mathrm{H}_{\mathbf{c}^{\prime}}\left(W^{\prime}, V\right) \simeq \mathrm{H}_{\mathbf{c}^{\prime}}\left(W^{\prime}, \bar{V}\right) \otimes \mathcal{D}\left(V^{W^{\prime}}\right)$ and

$$
\overline{\mathrm{H}}_{\mathbf{c}^{\prime}}\left(W^{\prime}, V\right) \simeq \overline{\mathrm{H}}_{\mathbf{c}^{\prime}}\left(W^{\prime}, \bar{V}\right) \otimes \overline{\mathcal{D}}\left(V^{W^{\prime}}\right),
$$

where $\overline{\mathcal{D}}\left(V^{W^{\prime}}\right):=\mathcal{D}\left(V^{W^{\prime}}\right) /\left\langle\left(k\left[\left(V^{W^{\prime}}\right)^{(1)}\right] \otimes k\left[\left(\left(V^{W^{\prime}}\right)^{*}\right)^{(1)}\right]\right)_{+}\right\rangle$. In particular, (2) and (3) are equivalent to

(2') $\mathrm{H}_{\mathbf{c}^{\prime}}\left(W^{\prime}, V\right)$ has no small modules $M$ with Supp $M=0$ for all parabolic subgroups $W^{\prime} \subseteq W$

(3') $\overline{\mathrm{H}}_{\mathbf{c}^{\prime}}\left(W^{\prime}, V\right)$ has no small modules $M$ for all parabolic subgroups $W^{\prime} \subseteq W$, respectively, where we have used the fact that $\mathcal{D}\left(V^{W^{\prime}}\right)$ is an Azumaya algebra. The equivalence of (1) and (2') is Corollary 5.3. Clearly (2') implies (3'). For the converse, suppose that $\mathrm{H}_{\mathbf{c}^{\prime}}\left(W^{\prime}, V\right)$ has a small module $M$ with $\operatorname{Supp} M=0$ for some parabolic subgroup $W^{\prime} \subseteq W$. Then by the proposition, $\operatorname{gr}_{\mathbb{Z}} M$ is a small module for $\overline{\mathrm{H}}_{\mathbf{c}^{\prime}}\left(W^{\prime}, V\right)$.

The equivalence of (3) and (4) follows from an identical argument to [17].

REMARK. The proofs above are valid, mutando mutandae, for $t=0$ and char $k=0$.

5.6. Smoothness of centres of $\mathrm{H}_{\mathbf{c}}(G(m, 1, n))$ Let $m, n$ be positive integers and assume that $n>1$. Let $W=G(m, 1, n)$. In this section we give a proof of Corollary 1.2. As noted at the beginning of this section, the idea is to use Corollary 5.5(4) together with the results of Section 4 to determine for which $\mathbf{c}$ the centre $Z_{\mathbf{c}}(W)$ is smooth. Analogous results for the rational Cherednik algebra associated to $G(m, 1, n)$ over $\mathbb{C}$ at $t=0$ are given in [18, Lemma 4.4] and, implicitly, in [26]. However, the techniques used there are completely different to the elementary approach given here. Recall that the parabolic subgroups of $W$ are of the form

$$
S_{k_{1}} \times \cdots \times S_{k_{t}} \times G\left(m, 1, n^{\prime}\right), \quad k_{1}+\cdots+k_{t}+n^{\prime} \leq n .
$$

Here $S_{k_{i}}$ denotes the symmetric group on $k_{i}$ letters, and $S_{0}=G(m, 1,0)=\{$ id $\}$ by definition. For such a parabolic subgroup, the representation $\bar{V}$ is the reflection 
representation. Recall that $\mathbf{c}=\left(\kappa, c_{1}, \ldots, c_{m-1}\right)$. Recall the parameters $a_{1}, \ldots, a_{m}$ from Theorem 4.4. Let $\mathcal{C}_{m, n}$ denote the set of all $\mathbf{c}$ such that either

$$
\kappa \in \mathbb{F}_{p}, \quad \text { or } \quad a_{i}-a_{j} \pm C \kappa \in \mathbb{F}_{p}
$$

for some $0 \leq i \neq j \leq m-1$ and integer $C$ such that $0 \leq C \leq n-1$. Thus, $\mathcal{C}_{m, n}$ is a finite union of hyperplanes in $k^{m}$.

THEOREM. The algebra $Z_{\mathbf{c}}(W)$ is smooth if and only if $\mathbf{c} \notin \mathcal{C}_{m, n}$.

Proof. Let us first suppose that $\mathbf{c} \in \mathcal{C}_{m, n}$. Recall that, for $\lambda \in \mathcal{P}(m, n)$,

$$
\operatorname{Res}^{\mathbf{a}} \lambda:=\sum_{i=0}^{m-1} x^{\left(a_{i}^{p}-a_{i}\right)} \operatorname{Res}_{\lambda^{i}}\left(x^{-\left(\kappa^{p}-\kappa\right)}\right) .
$$

Suppose that $\kappa \in \mathbb{F}_{p}$. Then $\kappa^{p}-\kappa=0$ and so $\operatorname{Res}^{\mathbf{a}}((n), \emptyset, \ldots, \emptyset)=\operatorname{Res}^{\mathbf{a}}\left((n)^{t}\right.$, $\emptyset, \ldots, \emptyset)$. Thus, by Theorem $4.4, \overline{\mathrm{H}}_{\mathbf{c}}(W, V)$ has a non-singleton block, and so Corollary 5.5 implies that $Z_{\mathbf{c}}(W)$ is singular. If $m>1$ and $a_{i}-a_{j}-C_{\kappa} \in \mathbb{F}_{p}$ for some $0 \leq C \leq n-1$, then

$$
a_{i}^{p}-a_{i}=a_{j}^{p}-a_{j}+C\left(\kappa^{p}-\kappa\right) .
$$

Without loss of generality, $i=1$ and $j=2$, and then

$$
\operatorname{Res}^{\mathbf{a}}((n), \emptyset, \emptyset, \ldots, \emptyset)=\operatorname{Res}^{\mathbf{a}}\left(\emptyset,\left(n-C, 1^{C}\right), \emptyset, \ldots, \emptyset\right)=x^{a_{1}^{p}-a_{1}} \sum_{i=0}^{n-1} x^{-i\left(\kappa^{p}-\kappa\right)}
$$

Thus, $\overline{\mathrm{H}}_{\mathbf{c}}(W, V)$ has a non-singleton block and $Z_{\mathbf{c}}(W)$ is singular. A similar argument applies in the case $-n+1 \leq C \leq 0$.

Now suppose that $\mathbf{c} \notin \overline{\mathcal{C}}_{m, n}$. We first show that $\overline{\mathrm{H}}_{\mathbf{c}}(W, V)$ has only singleton blocks. For a contradiction, suppose that there exist distinct $\lambda, \boldsymbol{\mu} \in \mathcal{P}(m, n)$ such that $\operatorname{Res}^{\mathrm{a}} \lambda=$ Res ${ }^{\mathrm{a}} \boldsymbol{\mu}$. Since $\kappa \notin \mathbb{F}_{p}, x^{-\left(\kappa^{p}-\kappa\right)} \neq 1$. Each box $b$ in the Young diagram of $\lambda^{i}$ contributes $x^{\left(a_{i}^{p}-a_{i}\right)} x^{-\operatorname{cont}(b)\left(\kappa^{p}-\kappa\right)}$ to Res ${ }^{\mathrm{a}} \lambda$. Since $\lambda \neq \mu$, there exist $1 \leq i<j \leq N$ and boxes $b \in \lambda^{i}$, $b^{\prime} \in \mu^{j}$ such that $x^{\left(a_{i}^{p}-a_{i}\right)} x^{-\operatorname{cont}(b)\left(\kappa^{p}-\kappa\right)}=x^{\left(a_{j}^{p}-a_{j}\right)} x^{-\operatorname{cont}\left(b^{\prime}\right)\left(\kappa^{p}-\kappa\right)}$. Now $\operatorname{cont}(b)$ and $\operatorname{cont}\left(b^{\prime}\right)$ are integers such that $-\left|\lambda^{i}\right|+1 \leq \operatorname{cont}(b) \leq\left|\lambda^{i}\right|-1$ and $-\left|\mu^{j}\right|+1 \leq \operatorname{cont}\left(b^{\prime}\right) \leq\left|\mu^{j}\right|-$ 1. Thus, $a_{i}^{p}-a_{i}=a_{j}^{p}-a_{j}+C\left(\kappa^{p}-\kappa\right)$ for some $-\left|\lambda^{i}\right|-\left|\mu^{i}\right|+1 \leq C \leq\left|\lambda^{i}\right|+\left|\mu^{i}\right|-1$. This means that $-a_{i}+a_{j}-C \kappa \in \mathbb{F}_{p}$, and so $\mathbf{c} \in \mathcal{C}_{m, n}$, a contradiction.

We now want to prove the stronger statement that $\mathbf{c} \notin \mathcal{C}_{m, n}$ implies that $Z_{\mathbf{c}}(W)$ is smooth. Note that $\mathcal{C}_{m^{\prime}, n^{\prime}} \subset \mathcal{C}_{m, n}$ for all $m^{\prime}=1$ or $m, n^{\prime}<n$. Therefore, $\notin \notin \mathcal{C}_{m, n}$ implies that $\mathbf{c}^{\prime} \notin \mathcal{C}_{m^{\prime}, n^{\prime}}$ for all $m^{\prime}=1$ or $m, n^{\prime}<n$. By the description of parabolic subgroups $W^{\prime} \subset W$ given above, the previous paragraph implies $\overline{\mathrm{H}}_{\mathbf{c}}\left(W^{\prime}, \bar{V}\right)$ has only singleton blocks for all $W^{\prime}$. By Corollary 5.5 this implies that $Z_{\mathbf{c}}(W)$ is smooth.

REMARK. (1) In the case $m=1, W$ is just the symmetric group $S_{n}$. Although $V=$ $k^{n}$ is not the reflection representation, we have $\mathrm{H}_{\kappa}\left(S_{n}, k^{n}\right) \cong \mathrm{H}_{\kappa}\left(S_{n}, k^{n-1}\right) \otimes \mathcal{D}\left(\mathbb{A}^{1}\right)$, where $k^{n-1}$ now denotes the reflection representation of $S_{n}$. The set $\mathcal{C}_{1, n}$ is then identified with $\mathbb{F}_{p} \subset k$.

(2) Note that the proof of the above theorem shows that for $W=G(m, 1, n)$, the centre $Z_{\mathbf{c}}(W)$ is smooth if and only if $\overline{\mathrm{H}}_{\mathbf{c}}(W, V)$ has only singleton blocks. 
6. Degenerations. The goal of this section is to give a proof of Theorem 1.3. Since the proof is rather long and technical, we outline here, for the reader's benefit, the basic idea. The key result is Lemma 6.7, which gives a formula for the Poincaré polynomial of the simple, graded $\overline{\mathrm{H}}_{\mathbf{c}}(W)$-module $L_{\mathbf{c}}(\lambda)$ under the assumption that $\operatorname{dim}_{k} L_{\mathbf{c}}(\lambda)=$ $p^{n}|W|$. Since $L_{\mathbf{c}}(\lambda)$ is finite-dimensional, this Poincaré polynomial must be a Laurent polynomial. However, the formula we derive is a rational function, which one can easily show is not a Laurent polynomial for certain choices of the representation $\lambda$. This contradicts our assumption that $\operatorname{dim}_{k} L_{\mathbf{c}}(\lambda)=p^{n}|W|$.

To illustrate the difficultly in deriving the formula given in Lemma 6.7, it is best to contrast it with the analogous result, [3, Lemma 3.3], for rational Cherednik algebras over $\mathbb{C}$ at $t=0$. In the latter situation, the baby Verma module $\bar{\Delta}(\lambda)$ is isomorphic to $\mathbb{C}[V]^{\operatorname{co} W} \otimes \lambda$ as a $\mathbb{C}[V] \rtimes W$-module. If the support of $L_{\mathbf{c}}(\lambda)$ is contained in the smooth locus of $Z_{\mathbf{c}}(W, V)$, then $L_{\mathbf{c}}(\lambda) \simeq \mathbb{C} W$ as a $W$-module, and all composition factors of $\bar{\Delta}(\lambda)$, as graded $\overline{\mathrm{H}}_{\mathbf{c}}(W)$-modules, are just graded shifts of $L_{\mathbf{c}}(\lambda)$. Therefore, as graded $W$-modules, we have $[\bar{\Delta}(\lambda)]=f(t)\left[L_{\mathbf{c}}(\lambda)\right]$ for some Laurent polynomial $f(t)$. Consider the occurrence of the trivial representation on both sides of this equation: $L_{\mathbf{c}}(\lambda)_{\text {triv }} \simeq \mathbb{C}$ which implies that $f(t)$ (up to a shift) is just the graded multiplicity of the trivial representation in $\mathbb{C}[V]^{\operatorname{co} W} \otimes \lambda$. It is easy to calculate this graded multiplicity.

However, in positive characteristic, $\bar{\Delta}(\lambda) \simeq k[V]^{p \operatorname{co} W} \otimes \lambda$, as a graded $k[V] \rtimes W$ module, and $L_{\mathbf{c}}(\lambda) \simeq(k W)^{\oplus p^{n}}$. We still get the same equation $[\bar{\Delta}(\lambda)]=f(t)\left[L_{\mathbf{c}}(\lambda)\right]$ in the appropriate Grothendieck group, but a priori have no idea what the grading is on the $p^{n}$-dimensional space $L_{\mathbf{c}}(\lambda)_{\text {triv }}$. What is required is a way to reduce the dimension of $L_{\mathbf{c}}(\lambda)$ from $p^{n}|W|$ to $|W|$. The way to do this is to use the DeRham functor, as introduced by Cartier. Firstly, we show that both $\bar{\Delta}(\lambda)$ and $L_{\mathbf{c}}(\lambda)$ form a flat family over the line $\ell$ through $\mathbf{c}$ and 0 in the parameter space $\mathcal{C}$. Therefore, we can specialize these modules at $\mathbf{c}=0$ to get $\mathrm{H}_{0}(W)=\mathcal{D}(V) \rtimes W$-modules $\bar{\Delta}_{0}(\lambda)$ and $L(\lambda)_{\mathbf{c}=0}$. The module $L(\lambda)_{\mathbf{c}=0}$ will no longer be irreducible but has same graded $W$-structure as $L_{\mathbf{c}}(\lambda)$. The module $\bar{\Delta}_{0}(\lambda)$ is equipped with a filtration such that each subquotient is isomorphic to a graded shift of $L(\lambda)_{\underline{\mathbf{c}}=0}$. Applying the DeRham functor to both $\bar{\Delta}_{0}(\lambda)$ and $L(\lambda)_{\underline{\mathbf{c}}=0}$ gives graded $k\left[V^{(1)}\right] \rtimes W$-modules $\bar{\Delta}_{0}(\lambda)^{(1)}$ and $L(\lambda)_{\mathbf{c}=0}^{(1)}$ say. These modules still satisfy the equation $\left[\bar{\Delta}_{0}(\lambda)^{(1)}\right]=f(t)\left[L(\lambda)_{\mathbf{c}=0}^{(1)}\right]$, but now $\operatorname{dim}_{k} L(\lambda)_{\mathbf{c}=0}^{(1)}=|W|$ and $L(\lambda)_{\mathbf{c}=0}^{(1)}$ is just a graded copy of the regular representation as a $W$-module. Similarly, $\bar{\Delta}_{0}(\lambda)^{(1)}$ is isomorphic to $k\left[V^{(1)}\right]^{\text {co } W} \otimes \lambda$. Thus, we are in a situation completely analogous to the $t=0$ over $\mathbb{C}$ situation described above and the proof of Lemma 6.7 is complete.

We begin by describing, based on Cartier's Theorem, the category of $\mathcal{D}(V) \rtimes W$ module with $p$-curvature zero.

6.1. $p$-curvature. Let $k$ be a field of characteristic $p>0, V$ a finite-dimensional $k$-vector space and $W \subset G L(V)$ a finite group. Fix a basis $x_{1}, \ldots, x_{n}$ of $V^{*}$ and $\partial_{1}, \ldots, \partial_{n}$ of $V$ such that $\partial_{i}\left(x_{j}\right)=\delta_{i, j}$. Let

$$
A=k\left[\partial_{1}, \ldots, \partial_{n}, x_{1}^{p}, \ldots, x_{n}^{p}\right] \text { and } \operatorname{Spec} A=T^{*,(1)} V .
$$

The centre of $\mathcal{D}(V)$ embeds in $A$ and we write $\pi: T^{*,(1)} V \rightarrow\left(T^{*} V\right)^{(1)}$ for the corresponding finite morphism. We require a special case, Proposition 6.1, of a classical result, [23, Theorem 5.1], attributed to Cartier, on $\mathcal{D}$-module with zero $p$-curvature. We follow the presentation given in [23, Section 5]. Recall that if $D \in \operatorname{Der}(V) \subset \mathcal{D}(V)$ 
is a derivation, then $D^{p}$ also acts as a derivation. We write $D^{[p]}$ for this derivation so that $D^{p}-D^{[p]}$ acts trivially on $k[V]$.

Definition. Let $M$ be a finitely generated $\mathcal{D}(V) \rtimes W$-module. The p-curvature of $M$ is the map $\psi: \operatorname{Der}(V) \rightarrow \operatorname{End}_{k}(M)$ given by $\psi(D)=\rho(D)^{p}-\rho\left(D^{[p]}\right)$, where $\rho$ : $\mathcal{D}(V) \rtimes W \rightarrow \operatorname{End}_{k}(M)$ is the action map. We say that $M$ has zero p-curvature if $\psi=0$.

Denote by $\mathcal{D}_{0}$ the full subcategory of $\mathcal{D}(V) \rtimes W$-mod consisting of modules with zero $p$-curvature.

Proposition. Let $M \in \mathcal{D}(V) \rtimes W$-mod. Let $V^{(1)} \subset\left(T^{*} V\right)^{(1)}$ be the zero section (defined by $\partial_{1}^{p}=\cdots=\partial_{n}^{p}=0$ ).

(1) The module $M$ has zero p-curvature if and only if $M$ is scheme-theoretically supported on $V^{(1)}$ when considered as a $Z(\mathcal{D}(V))$-module, i.e. $\partial_{i}^{p} \cdot M=0$ for all $i$.

(2) The 'horizontal sections' functor $D R: \mathcal{D}_{0} \rightarrow k\left[V^{(1)}\right] \rtimes W$-mod,

$$
D R(M):=M^{\nabla}=\left\{m \in M \mid \partial_{i} \cdot m=0 \forall i\right\},
$$

is an equivalence of categories with quasi-inverse $D R^{\perp}: k\left[V^{(1)}\right] \rtimes W$-mod $\rightarrow \mathcal{D}_{0}$,

$$
D R^{\perp}(N)=\operatorname{lnd}_{A \rtimes W}^{\mathcal{D}(V) \rtimes W} N,
$$

where $A$ acts on $N$ via the morphism $A \rightarrow k\left[V^{(1)}\right], \partial_{i} \mapsto 0$.

(3) The equivalence DR restricts to an equivalence of graded categories $\operatorname{gr} \mathcal{D}_{0} \rightarrow$ $k\left[V^{(1)}\right] \rtimes W$-grmod.

The following operators were introduced in [23, (5.1.2)]. Their properties can be verified by direct calculation.

Lemma. Let $\mathcal{D}(x)$ be the first Weyl algebra and $M$ be a $\mathcal{D}(x)$-module with zero $p$ curvature.

(1) Define $P=\sum_{i=0}^{p-1} \frac{(-x)^{i}}{i !} \partial^{i} \in \mathcal{D}(x)$. Then $P$ defines a $k\left[x^{p}\right]$-linear operator on $M$ such that $P(M) \subset M^{\nabla},\left.P\right|_{M^{\nabla}}=$ id and $P^{2}=P$.

(2) Define the map $T: M \rightarrow M$ by

$$
m \mapsto \sum_{i=0}^{p-1} \frac{x^{i}}{i !} P\left(\partial^{i} \cdot m\right)
$$

Then $T=\mathrm{id}_{M}$.

Proof of Proposition 6.1 It is shown in [23, Proposition 5.2] that $\psi$ is $p$-linear, i.e. $\psi\left(f_{1} D_{1}+f_{2} D_{2}\right)=f_{1}^{p} \psi\left(D_{1}\right)+f_{2}^{p} \psi\left(D_{2}\right)$ for all $f_{i} \in k[V]$ and $D_{i} \in \operatorname{Der}(V)$. Since $\psi\left(\partial_{i}\right)=$ $\rho\left(\partial_{i}\right)^{p}$ for all $i$, it is clearly necessary that $\rho\left(\partial_{i}\right)^{p}=0$, i.e. $\partial_{i}^{p} \cdot M=0$, in order for $M$ to have zero $p$-curvature. On the other hand, every element $D \in \operatorname{Der}(V)$ can be expressed as $D=\sum_{i=1}^{n} f_{i} \partial_{i}$ for some $f_{i} \in k[V]$; thus,

$$
\psi(D)=\sum_{i=1}^{n} f_{i}^{p} \partial_{i}^{p}
$$

and $\psi(D) \cdot M=0$ if $\partial_{i}^{p} \cdot M=0$ for all $i$. This proves statement (1). 
Now we prove statement (2). As in Lemma 6.1, define $P_{i}=\sum_{j=0}^{p-1} \frac{\left(-x_{i}\right)^{j}}{j !} \partial_{i}^{j}$ and set $P=\prod_{i=1}^{n} P_{i}$. Let $M \in \mathcal{D}_{0}$. Then Lemma 6.1 implies $P$ defines a $k\left[V^{(1)}\right]$-linear operator on $M$ such that $D R(M)=P(M), P_{M^{\nabla}}=$ id and $P^{2}=P$. Note that the subspace $M^{\nabla}$ of $M$ is a $W$-submodule of $M$. Therefore, we define

$$
\tilde{P}=\frac{1}{|W|} \sum_{w \in W} w \cdot P
$$

so that $\tilde{P}$ is a $W$-equivariant projection onto $M^{\nabla}$. Let

$$
0 \rightarrow M_{1} \rightarrow M_{2} \stackrel{\phi}{\rightarrow} M_{3} \rightarrow 0
$$

be a short exact sequence in $\mathcal{D}_{0}$. Since $\tilde{P} \in \mathcal{D}(V)$, we have $\tilde{P} \circ \phi=\phi \circ \tilde{P}$. Therefore, $D R(\phi): D R\left(M_{2}\right) \rightarrow D R\left(M_{3}\right)$ is surjective and the left exact functor $D R$ is actually exact. Similarly, since $\mathcal{D}(V) \rtimes W$ is flat over $A \rtimes W, D R^{\perp}$ is also an exact functor. If $\left(\partial_{1}, \ldots, \partial_{n}\right)$ is the left ideal of $\mathcal{D}(V) \rtimes W$ generated by $\partial_{1}, \ldots, \partial_{n}$, then $D R^{\perp}\left(k\left[V^{(1)}\right] \rtimes\right.$ $W)=\mathcal{D}(V) \rtimes W /\left(\partial_{1}, \ldots, \partial_{n}\right)$ and

$$
D R\left(\frac{\mathcal{D}(V) \rtimes W}{\left(\partial_{1}, \ldots, \partial_{n}\right)}\right)=\frac{A \rtimes W+\left(\partial_{1}, \ldots, \partial_{n}\right)}{\left(\partial_{1}, \ldots, \partial_{n}\right)} \simeq k\left[V^{(1)}\right] \rtimes W
$$

as a $k\left[V^{(1)}\right] \rtimes W$-module. Since $D R \circ D R^{\perp}\left(k\left[V^{(1)}\right] \rtimes W\right)=k\left[V^{(1)}\right] \rtimes W$ and the functor $D R \circ D R^{\perp}$ is exact, by choosing a finite presentation for each $N \in k\left[V^{(1)}\right] \rtimes$ $W$-mod, we get the standard diagram

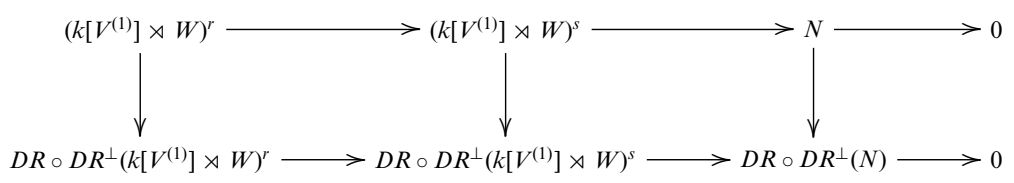

where the first two vertical morphisms are isomorphisms and the rows are exact. This implies that the third vertical morphism is also an isomorphism. Hence, the natural transformation $1 \rightarrow D R \circ D R^{\perp}$, coming from the fact that $D R^{\perp}$ is left adjoint to $D R$, is an isomorphism. Now take $M \in \mathcal{D}_{0}$ and consider the natural morphism $D R^{\perp} \circ D R(M) \rightarrow M$. Since $D R$ is conservative, the fact that $D R \circ D R^{\perp}=1$ implies that this morphism is injective. On the other hand, if we define $T=\prod_{i=1}^{n} T_{i}$, where $T_{i}: M \rightarrow M$,

$$
T_{i}(m)=\sum_{j=0}^{p-1} \frac{x_{i}^{j}}{j !} P_{i}\left(\partial_{i}^{j} \cdot m\right)
$$

then Lemma 6.1 implies that $T=\mathrm{id}_{M}$. This proves that $D R^{\perp} \circ D R(M) \rightarrow M$ is surjective.

In (3), it is straightforward to see that $D R$ and $D R^{\perp}$ send graded modules to graded modules.

6.2. Simple $\mathcal{D}(V) \rtimes W$-modules. We continue with the same setup as in the previous section, but assume now that $k$ is algebraically closed. A point ' $\zeta \in T^{*,(1)} V^{\text {' will }}$ 
therefore refer to a closed $k$-point. The group $W$ acts on $T^{*(1)} V$ and $\left(T^{*} V\right)^{(1)}$ such that the map $\pi$ is $W$-equivariant and satisfies

$$
W_{\zeta}:=\operatorname{Stab}_{W}(\zeta)=\operatorname{Stab}_{W}(\pi(\zeta)), \quad \forall \zeta \in T^{*,(1)} V .
$$

For fixed $\zeta \in T^{*,(1)} V$ and $\lambda \in \operatorname{Irr}_{k}\left(W_{\zeta}\right)$, we define $V_{\zeta}(\lambda):=\operatorname{Ind}_{A \rtimes W_{\zeta}}^{\mathcal{D}(V) W} \lambda$, where $A$ acts on $\lambda$ via the character $\zeta$.

LEMMA. Fix $\zeta \in T^{*,(1)} V$ and $\lambda \in \operatorname{Irr}_{k}\left(W_{\zeta}\right)$. Then

(1) the $\mathcal{D}(V) \rtimes W$-module $V_{\zeta}(\lambda)$ is simple;

(2) $V_{\zeta_{1}}\left(\lambda_{1}\right) \simeq V_{\zeta_{2}}\left(\lambda_{2}\right)$ if and only if $\zeta_{2} \in W \cdot \zeta_{1}$ and, moreover, if $w \zeta_{1}=\zeta_{2}$ then $\lambda_{1} \simeq$ $\lambda_{2}$ via the conjugation isomorphism $w: W_{\zeta_{1}} \stackrel{\sim}{\longrightarrow} W_{\zeta_{2}}$;

(3) every simple $\mathcal{D}(V) \rtimes W$-module is isomorphic to $V_{\zeta}(\lambda)$ for some $\zeta$ and $\lambda$.

Proof. Considered as an $A$-module, $V_{\zeta}(\lambda)=\bigoplus_{\eta \in W \cdot \zeta}\left(V_{\zeta}(\lambda)\right)_{\eta}$, where $\left(V_{\zeta}(\lambda)\right)_{\eta}$ is set-theoretically supported at $\eta$. Each $\left(V_{\zeta}(\lambda)\right)_{w \zeta}$ is a $\mathcal{D}(V) \rtimes\left(w W_{\zeta} w^{-1}\right)$-submodule of $V_{\zeta}(\lambda)$, and $V_{\zeta}(\lambda)$ will be a simple $\mathcal{D}(V) \rtimes W$-module if and only if $\left(V_{\zeta}(\lambda)\right)_{\zeta}=$ Ind ${ }_{A \rtimes W_{\zeta}}^{\mathcal{D}(V) \rtimes W_{\zeta}} \lambda$ is a simple $\mathcal{D}(V) \rtimes W_{\zeta}$-module. If $\zeta=(a, \alpha)$ with $a \in V^{(1)}$, then we write $(b, \alpha)$ with $b \in V$ for the unique closed point in $\pi^{-1}(\zeta)$. Applying the $W_{\zeta}$-equivariant automorphism $x_{i} \mapsto x_{i}-\left\langle x_{i}, b\right\rangle$ and $\partial_{j} \mapsto \partial_{j}-\left\langle\partial_{j}, \alpha\right\rangle$ to $\mathcal{D}(V)$, we may assume without loss of generality that $\zeta=0$ and $W_{\zeta}=W$. Let $\delta^{0}=$ Ind $_{A}^{\mathcal{D}(V)} k$ be the unique simple $\mathcal{D}(V)$-module supported at $0 \in\left(T^{*} V\right)^{(1)}$; simplicity of $\delta^{0}$ follows from the fact that $\mathcal{D}(V)$ is Azumaya of rank $p^{2 n}$ and the dimension of $\delta^{0}$ is $p^{n}$. Then $V_{0}(\lambda)=\delta^{0} \otimes \lambda$, with $W$ acting diagonally. The module $V_{0}(\lambda)$ is simple: let $v_{1}, \ldots, v_{p^{n}}$ be a basis of $\delta^{0}$ such that $v_{1}=1$ and choose any $0 \neq l=\sum_{i} v_{i} \otimes l_{i} \in V_{0}(\lambda)$. Choose an $i$ such that $l_{i} \neq 0$. Since $\mathcal{D}(V)$ surjects onto $\operatorname{End}_{k}\left(\delta^{0}\right)$, there is some $D \in \mathcal{D}(V)$ such that $D \cdot v_{j}=0$ for all $j \neq i$ and $D \cdot v_{i}=v_{1}$. Then $D \cdot l=1 \otimes l_{i}$ and we have $W \cdot(D \cdot l)=1 \otimes \lambda$. Hence, $\mathcal{D}(V) \rtimes W \cdot l=V_{0}(\lambda)$. To show that the various $V_{0}(\mu)$ are non-isomorphic, note that

$$
\operatorname{dim}_{k} \operatorname{Hom}_{\mathcal{D}(V) \rtimes W}\left(V_{0}(\lambda), V_{0}(\mu)\right)=\operatorname{dim}_{k} \operatorname{Hom}_{A \rtimes W}\left(\lambda, V_{0}(\mu)\right)=\delta_{\lambda, \mu}
$$

because the space $\left\{v \in \delta^{0} \mid \partial_{i} \cdot v=0 \forall i\right\}$ is one-dimensional. Arguing geometrically as above shows the second claim of the statement. The final statement is clear just by considering the socle, as a $A$-module, of a simple $\mathcal{D}(V) \rtimes W$-module.

6.3. The regular representation. Let 1 denote the trivial $W$-module. The following observation will be required later.

LeMmA. Assume that $p$ is prime to the order of $W$. Let $N$ be a $k\left[V^{(1)}\right] \rtimes W$-module such that $D R^{\perp}(N)$ is isomorphic to $p^{n}$ copies of the regular representation as a $W$-module. Then $N$ is isomorphic to the regular representation as a $W$-module.

Proof. As a $W$-module, $D R^{\perp}(N) \simeq V_{0}(\mathbf{1}) \otimes N$. Therefore, it suffices to show that the Brauer character $\chi$ of $V_{0}(\mathbf{1})$ satisfies $\chi(w) \neq 0$ for all $w$ in $W$. Recall that $V_{0}(\mathbf{1}) \simeq k\left[V^{*}\right] /\left\langle k\left[\left(V^{*}\right)^{(1)}\right]_{+}\right\rangle$. Let $\lambda_{1}, \ldots, \lambda_{n}$ be the eigenvalues of $w$ on $V$. Since we are calculating the Brauer character of $V_{0}(\mathbf{1})$, we assume that $\lambda_{i} \in \mathbb{C}$ for all $i$. Then

$$
\chi(g)=\operatorname{Tr}\left(g, V_{0}(\mathbf{1})\right)=\prod_{i=1}^{n} \frac{1-\lambda_{i}^{p}}{1-\lambda_{i}} .
$$


Since $\chi(g) \in \mathbb{C}$ and $p$ does not divide $|W|$, the product on the right-hand side is nonzero.

6.4. Lattices. In this section let $k$ be an arbitrary algebraically closed field of characteristic $\geq 0$. We assume this level of generality since the results of this section may be of use in the study of restricted rational Cherednik algebras at $t=0$ over $\mathbb{C}$. Let $H$ denote a $\mathbb{Z}$-graded $k[x]$-algebra such that $H$ is a finite, free $k[x]$-module, where $k[x]$ is graded with $\operatorname{deg}(x)=0$. Write $\ell=\operatorname{Spec} k[x]$ and $K=k(x)$. For $\alpha \in k$, we denote by $H_{\alpha}$ the specialization $H \otimes_{k[x]} k_{\alpha}$ of $H$ at $\alpha$. We assume that there exists a finite set $I$ and $H$-modules $\{\Delta(\lambda) \mid \lambda \in I\}$ such that

(1) the module $\Delta(\lambda)$ is graded and free as a $k[x]$-module.

(2) For all $\alpha \in \ell$, there is a bijection $\operatorname{Irr} H_{\alpha} \simeq I$ such that the simple, graded $H_{\alpha}$-module $L_{\alpha}(\lambda)$, for $\lambda \in I$, is the unique simple quotient of $\Delta_{\alpha}(\lambda)$, the specialization of $\Delta(\lambda)$ at $\alpha$.

(3) There is a bijection $I \simeq \operatorname{Irr} H_{K}$ such that the simple, graded $H_{K}$-module $L_{K}(\lambda)$ is the unique simple quotient of $\Delta_{K}(\lambda)$.

Let $M$ be a $H$-module. We say that $M$ is a $H$-lattice if it is free, of finite rank, as a $k[x]$-module. Note that if $M$ is a $H$-lattice and $N$ is a $H$-submodule, then $N$ is a $H$-lattice because $k[x]$ is a principal ideal domain.

LEMMA. There exists a composition series $0=\Delta^{0}(\lambda) \subset \cdots \subset \Delta^{r}(\lambda)=\Delta(\lambda)$ of graded $H$-lattices such that if $L^{i}(\lambda)=\Delta^{i}(\lambda) / \Delta^{i-1}(\lambda)$, then $L^{i}(\lambda)$ is a graded $H$-lattice and $L^{i}(\lambda)_{K} \simeq L_{K}\left(\lambda_{i}\right)$ for some $\lambda_{i} \in I$.

Proof. Fix a graded composition series

$$
0=\Delta_{K}^{0}(\lambda) \subset \cdots \subset \Delta_{K}^{r}(\lambda)=\Delta_{K}(\lambda)
$$

such that $\Delta_{K}^{i}(\lambda) / \Delta_{K}^{i-1}(\lambda) \simeq L_{K}\left(\lambda_{i}\right)$ for some $\lambda_{i} \in I$. Write $\phi: \Delta(\lambda) \rightarrow \Delta_{K}(\lambda)$ for the natural map. It is an inclusion. We set

$$
\Delta^{i}(\lambda)=\phi^{-1}\left(\Delta_{K}^{i}(\lambda)\right)=\Delta_{K}^{i}(\lambda) \cap \Delta(\lambda), \quad \forall i .
$$

Then $\Delta^{i}(\lambda)$ is a $H$-submodule of the $H$-lattice $\Delta(\lambda)$, hence it is a $H$-lattice. We have a $H$-morphism $\phi_{i}: L^{i}(\lambda):=\Delta^{i}(\lambda) / \Delta^{i-1}(\lambda) \rightarrow \Delta_{K}^{i}(\lambda) / \Delta_{K}^{i-1}(\lambda)=L_{K}\left(\lambda_{i}\right)$. We claim that $L^{i}(\lambda)$ is a $H$-lattice. It suffices to show that it is torsion-free with respect to $k[x]$. Let $\bar{a} \in$ $L^{i}(\lambda)$ and $0 \neq f(x) \in k[x]$ such that $f(x) \cdot \bar{a}=0$. Then $f \cdot a \in \Delta^{i-1}(\lambda)=\Delta_{K}^{i-1}(\lambda) \cap \Delta(\lambda)$, which implies that $a \in \Delta_{K}^{i-1}(\lambda) \cap \Delta(\lambda)=\Delta^{i-1}(\lambda)$. Hence, $\bar{a}=0$. Since $L^{i}(\lambda)$ is a $H$ lattice and $L_{K}\left(\lambda_{i}\right)$ is a simple $H_{K}$-module, $\phi_{i}$ will induce an isomorphism $L^{i}(\lambda)_{K} \simeq$ $L_{K}\left(\lambda_{i}\right)$, provided $L^{i}(\lambda) \neq 0$. Let $0 \neq b \in \Delta_{K}^{i}(\lambda)-\Delta_{K}^{i-1}(\lambda)$, then there exists some $0 \neq$ $g(x) \in k[x]$ such that $g(x) b \in \Delta_{K}^{i}(\lambda) \cap \Delta(\lambda)$ and $g(x) b \notin \Delta_{K}^{i-1}(\lambda)$. Therefore, $g(x) b \in$ $\Delta^{i}(\lambda)-\Delta^{i-1}(\lambda)$.

6.5. Deformations. By Lemma 6.4 , for each $\lambda \in I$ we have a graded $H$-lattice $L(\lambda):=$ $L^{r}(\lambda)$ such that $\Delta(\lambda) \rightarrow L(\lambda)$. The specialization of $L(\lambda)$ to $x=\alpha \in k$ is denoted by $L(\lambda)_{x=\alpha}$ to distinguish it from the simple module $L_{\alpha}(\lambda)$.

LEMMA. There exists a prime ideal $\mathfrak{p} \triangleleft H$ such that $\mathfrak{p} \cdot L(\lambda)=0$.

Proof. Let $\mathrm{Ann}_{1}=\{I \triangleleft H \mid \operatorname{GKdim}(\Delta(\lambda) / I \Delta(\lambda))=1\}$. Since $\operatorname{GKdim}(\Delta(\lambda))=1$, the set $\mathrm{Ann}_{1}$ is non-empty. Since $H$ is Noetherian, we can choose $\mathfrak{p} \in \mathrm{Ann}_{1}$ to be 
maximal with respect to inclusion. The claim is that $\mathfrak{p}$ is prime. Assume otherwise so that there exist ideals $I, J$ with $I J \subset \mathfrak{p}$ but $I, J \notin \mathrm{Ann}_{1}$. Note that $\Delta / \mathfrak{p} \cdot \Delta$ has GK-dimension one and is a finitely generated $H / \mathfrak{p}$-module. Therefore, $H / \mathfrak{p}$ has GKdimension one too. Since $\Delta / J \Delta$ has GK-dimension zero, the short exact sequence

$$
0 \rightarrow J \Delta / \mathfrak{p} \Delta \rightarrow \Delta / \mathfrak{p} \Delta \rightarrow \Delta / J \Delta \rightarrow 0
$$

implies, by [29, Proposition 8.3.11], that $\operatorname{GKdim}(J \Delta / \mathfrak{p} \Delta)=1$. However, $J \Delta / \mathfrak{p} \Delta$ is clearly a torsion $H / \mathfrak{p}$-module. Therefore, [29, Corollary 8.3.6] implies that the GKdimension of $J \Delta / \mathfrak{p} \Delta$ is zero. This contradiction implies that $\mathfrak{p}$ is prime. Let $M=$ $\Delta / \mathfrak{p} \cdot \Delta$. The $H$-submodule of $M$ consisting of elements that are torsion with respect to $k[x]$ is a proper submodule of GK-dimension zero. Therefore, quotienting out by this submodule we may assume that $M$ is torsion-free and hence free, and that $\mathfrak{p} \cdot M=0$. Moreover, $M_{K}$ is a non-zero quotient of $\Delta_{K}(\lambda)$, which implies that $M_{K} \rightarrow L_{K}(\lambda)$. This implies that $\mathfrak{p} \cdot L_{K}(\lambda)$. Since $L(\lambda)$ is a $H$-submodule of $L_{K}(\lambda)$, we have $\mathfrak{p} \cdot L(\lambda)=0$ as required.

Proposition. Let $m_{\lambda}=\max _{\alpha \in \ell}\left(\operatorname{dim}_{k} L_{\alpha}(\lambda)\right)$.

(1) There exists a finite set $\ell_{0} \subset \ell$ such that $\operatorname{dim}_{k} L_{\alpha}(\lambda)=m_{\lambda} \Leftrightarrow \alpha \notin \ell_{0}$.

(2) The rank of $L(\lambda)$ equals $m_{\lambda}$.

(3) The specialization of $L(\lambda)$ at $\alpha$ is isomorphic to the simple $H_{\alpha}$-module $L_{\alpha}(\lambda)$ for all $\alpha \in \ell-\ell_{0}$.

Proof. Let $\mathfrak{p}$ be a prime ideal in $H$ such that $\mathfrak{p} \cdot L(\lambda)=0$. Its existence is guaranteed by Lemma 6.5. Set $R:=H / \mathfrak{p}$ so that $L(\lambda)$ is an $R$-module. Since $R$ is a prime ring, the image of $k[x]$ in $R$ is a domain. Therefore, the fact that $R$ is a finite $k[x]$-module of GKdimension one implies that $R$ is a free $k[x]$-module. The map $\pi: \operatorname{Spec} Z(R) \rightarrow \operatorname{Spec} k[x]$ is finite. Let $\mathcal{A}$ denote the Azumaya locus of $R$. Since $\mathcal{A}$ is open and dense in the irreducible variety $\operatorname{Spec} Z(R)$ and the map $\pi$ is finite, the set $\pi(\operatorname{Spec} Z(R)-\mathcal{A})$ is a proper closed subset of $\ell$. Therefore, the set

$$
\ell_{1}:=\ell-\pi(\operatorname{Spec} Z(R)-\mathcal{A})=\left\{\alpha \in \ell \mid \pi^{-1}(\alpha) \subset \mathcal{A}\right\}
$$

is open and dense in $\ell$. Since the specialization $L(\lambda)_{x=\alpha}$ is a quotient of $\Delta(\lambda)_{x=\alpha}=$ $\Delta_{\alpha}(\lambda)$, the simple module $L_{\alpha}(\lambda)$ is a quotient of $L(\lambda)_{x=\alpha}$. This implies that every $L_{\alpha}(\lambda)$ is an $R$-module and hence $m_{\lambda}$ is bounded above by the PI degree of $R$. By definition, this bounded is achieved for all $\alpha \in \ell_{1}$. Fix some $\alpha \in \ell_{1}$. Recall that $L_{K}(\lambda)=K \cdot L(\lambda)$. Hence,

$$
\operatorname{dim}_{K} L_{K}(\lambda)=\operatorname{rank}_{k[x]} L(\lambda) \geq \operatorname{dim}_{k} L_{\alpha}(\lambda) .
$$

On the other hand, since $L_{\alpha}(\lambda)$ is supported on the Azumaya locus of $R$ and $L_{K}(\lambda)$ is a simple module for the central localization $R_{K}$ of $R$, [29, Posner's Theorem 13.6.5], together with [29, Kaplansky's Theorem 13.3.8], implies that

$$
\operatorname{dim}_{k}\left(L_{\alpha}(\lambda)\right)=\text { P.I. }-\operatorname{degree}(R) \geq \operatorname{dim}_{K} \operatorname{dim}_{K} L_{K}(\lambda)=\operatorname{rank}_{k[x]} L(\lambda) .
$$

Therefore, we get the required equality.

6.6. The restricted Cherednik algebra. We return to the case where $k$ is algebraically closed to characteristic $p>0$ and we assume that $p$ is prime to the order of $W$, a 
pseudo-reflection group. Recall that $\mathcal{C}$ is the space of parameters for $\mathrm{H}$. We fix a parameter c. Let $\underline{\mathbf{c}}$ be a formal variable. We denote by $\mathrm{H}_{\mathbf{c}}(W)$ the rational Cherednik algebra defined over $k[\mathbf{c}]$ such that the specialization of $\bar{H}_{\underline{\mathbf{c}}}(W)$ at $\underline{\mathbf{c}}=1$ is isomorphic to $\mathrm{H}_{\mathbf{c}}(W)$. Let $K=k(\underline{\mathbf{c}})$ be the field of fractions of $k[\underline{\mathbf{c}}]$. Given $\lambda \in \operatorname{Irr}_{k}(W)$, let $\bar{\Delta}_{\underline{\mathbf{c}}}(\lambda)=$ $\overline{\mathrm{H}}_{\underline{\mathbf{c}}}(W) \otimes_{k[V] \rtimes W} \lambda$ be the corresponding baby Verma module. It is a free $k[\mathbf{c}]$-module of finite rank. Let $\bar{\Delta}_{K}(\lambda)=\overline{\mathrm{H}}_{K}(W) \otimes_{K[V] \rtimes W} K \otimes_{k} \lambda$ be the corresponding baby Verma module for $\overline{\mathrm{H}}_{K}(W)$.

LEMMA. (1) Every simple module $\lambda \in \operatorname{Irr}_{K}(W)$ is absolutely irreducible.

(2) The module $\bar{\Delta}_{K}(\lambda)$ has a unique simple quotient $L_{K}(\lambda)$.

(3) We have a natural identification of $\overline{\mathrm{H}}_{K}(W)$-modules $K \otimes_{k[\mathbf{c}]} \bar{\Delta}_{\underline{\mathbf{c}}}(\lambda)=\bar{\Delta}_{K}(\lambda)$.

Proof.

(1) The $k$-algebra $k W$ is already split semi-simple since $k$ is assumed to be algebraically closed and the characteristic of $k$ does not divide the order of $|W|$. Therefore, the $K$-algebra $K W=K \otimes_{k} k W$ is also split semi-simple.

(2) Let $\mu \in \operatorname{Irr}_{K}(W)$, considered as a $K[V] \rtimes W$-module such that $K[V]_{+}$acts as zero. Since $K \lambda$ and $\mu$ are absolutely irreducible, adjunction implies that

$$
\operatorname{Hom}_{K[V] \rtimes W}\left(\bar{\Delta}_{K}(\lambda), \mu\right)=\operatorname{Hom}_{W}(K \lambda, \mu)=K \delta_{\lambda, \mu} .
$$

Therefore, if $\phi: \bar{\Delta}_{K}(\lambda) \rightarrow S$ is any simple quotient of $\bar{\Delta}_{K}(\lambda)$, we must have a $K[V] \rtimes W$-surjection $S \rightarrow K \lambda$. Hence, $S=\overline{\mathrm{H}}_{K}(W) \cdot K \lambda$ is the unique graded quotient of $\bar{\Delta}_{K}(\lambda)$.

(3) The subspace $K \otimes_{k} \lambda$ of $K \otimes_{k[\underline{c}]} \bar{\Delta}_{\underline{\mathbf{c}}}(\lambda)$ is a $K[V] \rtimes W$-submodule such that $K[V]_{+}$acts as zero. Moreover, this subspace generates $K \otimes_{k[\mathbf{c}]} \bar{\Delta}_{\underline{\mathbf{c}}}(\lambda)$ as a $\overline{\mathrm{H}}_{K}(W)$-module. Therefore, there exists a surjective morphism of $\overline{\mathrm{H}}_{K}(W)$ modules $\bar{\Delta}_{K}(\lambda) \rightarrow K \otimes_{k[\mathfrak{c}]} \bar{\Delta}_{\underline{\mathbf{c}}}(\lambda)$. Since both modules have the same dimension over $K$, this surjection must $\bar{b}$ e an isomorphism.

6.7. The proof of Theorem 1.3. As noted at the beginning of this section, the key to proving Theorem 1.3 is Lemma 6.7. The proof of this lemma uses the results of all the previous subsections of Section 6. Let ev : $K(W)\left[t, t^{-1}\right] \rightarrow \mathbb{Z}\left[t, t^{-1}\right]$ be the map sending $[\lambda]$ to $\operatorname{dim} \lambda$. Let

$$
I(t):=\operatorname{ev}\left(\operatorname{ch}_{t, W}\left(V_{0}(\mathbf{1})\right)\right)=\left(\frac{1-t^{p}}{1-t}\right)^{n} .
$$

Lemma. If $\mathbf{c} \in \mathcal{C}$ such that $\operatorname{dim}_{k} L_{\mathbf{c}}(\lambda)=|W| p^{n}$, then the Poincare polynomial of $L_{\mathbf{c}}(\lambda)$ is given by

$$
P\left(L_{\mathbf{c}}(\lambda), t\right)=\frac{\operatorname{dim}(\lambda) \cdot t^{p b_{\lambda^{*}}} P\left(k[V]^{c o W}, t^{p}\right) \cdot I(t)}{f_{\lambda^{*}}\left(t^{p}\right)} .
$$

Proof. Let $L(\lambda)$ be the $\mathrm{H}_{\underline{\mathbf{c}}}(W)$-module described in Section 6.5. Since it is free as a $k[\mathbf{c}]$-module, the graded $W$-module structure of all specializations of $L(\lambda)$ are the same. Therefore, it suffices to prove that the Poincare polynomial of the specialization $L(\lambda)_{\mathbf{c}=0}$ has the desired form. We fix a filtration $\bar{\Delta}_{\underline{\mathbf{c}}}^{i}(\lambda)$ of $\bar{\Delta}_{\underline{\mathbf{c}}}(\lambda)$ as in Lemma 6.4. Specializing 
to $\underline{\mathbf{c}}=0$ gives a filtration $\bar{\Delta}_{0}^{i}(\lambda)$ of $\bar{\Delta}_{0}(\lambda)$ such that $\bar{\Delta}_{0}^{r}(\lambda) / \bar{\Delta}_{0}^{r-1}(\lambda) \simeq L(\lambda)_{\underline{c}=0}$. All the $\mathcal{D}(V) \rtimes W$-modules $\bar{\Delta}_{0}^{i}(\lambda)$ have $p$-curvature zero. Therefore, Proposition 6.1 says that $\bar{\Delta}_{0}^{i}(\lambda)=\operatorname{Ind}_{A \rtimes W}^{\mathcal{D}(V) \rtimes W} \bar{\Delta}_{0}^{i}(\lambda)^{(1)}$ for some graded $k\left[V^{(1)}\right] \rtimes W$-module $\bar{\Delta}_{0}^{i}(\lambda)^{(1)}$ and we have

$$
\bar{\Delta}_{0}^{r}(\lambda)^{(1)} / \bar{\Delta}_{0}^{r-1}(\lambda)^{(1)}:=L(\lambda)_{\underline{\mathbf{c}}=0}^{(1)}, \quad \text { with } \quad L(\lambda)_{\underline{\mathbf{c}}=0}=\operatorname{Ind}_{A \rtimes W}^{\mathcal{D}(V) \rtimes W} L(\lambda)_{\underline{\mathbf{c}}=0}^{(1)} .
$$

This implies that $L_{\mathbf{c}}(\lambda) \simeq V_{0}(\mathbf{1}) \otimes L_{0}(\lambda)^{(1)}$ as graded $W$-modules. Let $\overline{\mathrm{H}}_{\mathbf{c}}(W)$-latt denote the category of $\overline{\mathrm{H}}_{\mathbf{c}}(W)$-lattices, i.e. the category of finitely generated, graded $\overline{\mathrm{H}}_{\mathbf{c}}(W)$-modules that are free over $k[\mathbf{c}]$. It is an exact, extension closed subcategory of $\overline{\mathrm{H}}_{\mathrm{c}}(W)$-grmod. Therefore, it makes sense to consider the Grothendieck group $K_{0}\left(\overline{\mathrm{H}}_{\mathbf{c}}(W)\right.$-latt $)$ of $\overline{\mathrm{H}}_{\mathbf{c}}(W)$-latt. By our assumption on $\operatorname{dim}_{k} L_{\mathfrak{c}}(\lambda)$, the support of $L_{\mathfrak{c}}(\lambda)$ as a $\bar{Z}_{\mathbf{c}}(W)$-module is contained in the Azumaya locus of $\mathrm{H}_{\mathbf{c}}(W)$. Therefore, Müller's Theorem, [8, Proposition 2.7], implies that each graded composition factor of the indecomposable module $\bar{\Delta}_{\mathfrak{c}}(\lambda)$ is of the form $L_{\mathfrak{c}}(\lambda)\left[m_{i}\right]$ for some integer $m_{i}$. Hence, Lemma 6.4 says that each $L_{\underline{\mathbf{c}}}^{i}(\lambda)$ specializes at $\underline{\mathbf{c}}=1$ to some $L_{\mathbf{c}}(\lambda)\left[m_{i}\right]$. Thus, in $K_{0}\left(\overline{\mathrm{H}}_{\underline{\mathbf{c}}}(W)\right.$-latt) we have an equality $\left[\bar{\Delta}_{\underline{\mathbf{c}}}(\lambda)\right]=h(t)\left[L_{\mathfrak{c}}(\lambda)\right]$ for some Laurent polynomial $h(t)$. This implies that $\left[\bar{\Delta}_{0}(\lambda)^{(1)}\right]=h(t)\left[L(\lambda)_{\mathbf{c}=0}^{(1)}\right]$ in the Grothendieck group of graded $k\left[V^{(1)}\right] \rtimes W$-modules. By Lemma 6.3, the fact that $L(\lambda)_{\underline{\mathbf{s}}=0}$ is isomorphic to $p^{n}$ copies of the regular representation implies that $L(\lambda)_{\mathbf{c}=0}^{(1)}$ is isomorphic to a graded copy of the regular representation. On the other hand, Lemma 2.3 and Proposition 6.1 imply that $D R^{\perp}\left(\bar{\Delta}_{0}(\lambda)\right) \simeq k\left[V^{(1)}\right]^{\mathrm{co} W} \otimes \lambda$, hence

$$
\left[k\left[V^{(1)}\right]^{\operatorname{co} W} \otimes \lambda\right]=h(t)\left[L(\lambda)_{\underline{\underline{c}}=0}^{(1)}\right] .
$$

The proof of [3, Lemma 3.3] implies that $h(t)=t^{-p b_{\lambda^{*}}} f_{\lambda^{*}}\left(t^{p}\right)$. From this, one can deduce formula (18).

Now we are finally in a position to prove Theorem 1.3.

Proof of Theorem 1.3 Assume that $W$ and $\mathbf{c}$ are chosen so that the centre of $\mathrm{H}_{\mathbf{c}}(W)$ is smooth. Then every simple $\mathrm{H}_{\mathbf{c}}(W)$-module has dimension $|W| p^{n}$. This implies that the graded character of $L_{\mathfrak{c}}(\lambda)$ is given by the formula of Lemma 6.7. Since $L_{\mathfrak{c}}(\lambda)$ is finitedimensional, the rational function on the right-hand side of (18) must be a Laurent polynomial. This means that $f_{\lambda^{*}}\left(t^{p}\right)$ divides $P\left(k[V]^{c o W}, t^{p}\right) I(t)$ in $\mathbb{C}\left[t, t^{-1}\right]$. Formula (17) for $I(t)$ shows that every root of $I$ is a primitive $p$ th root of unity. Therefore, if $f_{\lambda^{*}}\left(t^{p}\right)$ and $I(t)$ have a non-trivial common factor, we must have $f_{\lambda^{*}}\left(\zeta^{p}\right)=0$ for some primitive $p$ th root of unity $\zeta$. But $f_{\lambda^{*}}(1)=\operatorname{dim} \lambda^{*} \neq 0$. Hence, $f_{\lambda^{*}}\left(t^{p}\right)$ must divide $P\left(k[V]^{\text {co } W}, t^{p}\right)$ in $\mathbb{C}\left[t^{p}, t^{-p}\right]$. It was shown in [3] that if $W$ is not isomorphic to $G(m, 1, n)$ or $G_{4}$, then one can always find some $\lambda$ for which $f_{\lambda^{*}}\left(t^{p}\right)$ does not divide $P\left(k[V]^{\text {coW } W}, t^{p}\right)$. Since $\mathbf{c}$ has played no part in this argument, we conclude that the centre of $\mathrm{H}_{\mathbf{c}}(W)$ is never smooth in these cases.

Conversely, it follows from Corollary 1.2 that the centre of $\mathrm{H}_{\mathbf{c}}(G(m, 1, n))$ is regular for generic $\mathbf{c}$. Now we consider the group $G_{4}$; we have $p \neq 2,3$ in this case. By Corollary 5.5 , it suffices to show that the blocks of $\overline{\mathrm{H}}_{\mathbf{c}^{\prime}}\left(W^{\prime}\right)$ are singletons for generic parameters c and all parabolic subgroups $W^{\prime}$ of $G_{4}$. We begin by showing that this is true for $W^{\prime}=$ $G_{4}$. Recall from Section 3.1 the central element $\mathbf{h}^{p}-\mathbf{h}$ of $\mathrm{H}_{\mathbf{c}}\left(G_{4}\right)$. For the remainder of the proof we follow the notation of [3, Section 4]. The function $\mathbf{c}$ is defined by $\mathbf{c}\left(s_{i}\right)=c_{1}$ and $\mathbf{c}\left(t_{i}\right)=c_{2}$ for some $c_{1}, c_{2} \in k$. To show that the block partition of $\operatorname{Irr} G_{4}$ is trivial, it 
suffices to show that the scalars by which the element $\mathbf{h}^{p}-\mathbf{h}$ acts on $L(\lambda)$ 's are pairwise distinct. Let $z_{1}=s_{1}+\cdots+s_{4}$ and $z_{2}=t_{1}+\cdots+t_{4} \in Z\left(G_{4}\right)$. The scalar $\mu_{i}$ by which the central element $z_{i}$ acts on the simple $G_{4}$-module $\mu$ is given by

\begin{tabular}{c|cc}
$\mu$ & $z_{1}$ & $z_{2}$ \\
\hline$T$ & 4 & 4 \\
$V_{1}$ & $4 \omega^{2}$ & $4 \omega$ \\
$V_{2}$ & $4 \omega$ & $4 \omega^{2}$ \\
$W$ & -2 & -2 \\
$\mathfrak{h}$ & $-2 \omega^{2}$ & $-2 \omega$ \\
$\mathfrak{h}^{*}$ & $-2 \omega$ & $-2 \omega^{2}$ \\
$U$ & 0 & 0
\end{tabular}

We have $1-\lambda_{s_{i}}=1-\omega^{2}$ and $1-\lambda_{t_{i}}=1-\omega$. Define $d_{i}=\frac{-c_{i}}{1-\lambda_{i}} \in k$. Then $\mathbf{h}^{p}-\mathbf{h}$ acts on $L(\mu)$ by $d_{1}^{p} \mu_{1}^{p}-d_{1} \mu_{1}+d_{2}^{p} \mu_{2}^{p}-d_{2} \mu_{2}$, hence it will act by the same scalar on $L(\mu)$ and $L(\rho)$ if and only if

$$
d_{1}\left(\mu_{1}-\rho_{1}\right)+d_{2}\left(\mu_{2}-\rho_{2}\right) \in \mathbb{F}_{p}
$$

Since $|k|=\infty$ and the list of values $\mu_{i}-\rho_{i}$ is finite, we can always choose $d_{1}, d_{2} \in k$ such that (19) does not hold, provided there is no pair $\mu \neq \rho$ such that $\mu_{1}=\rho_{1}$ and $\mu_{2}=\rho_{2}$. This can be checked directly, e.g. $-2=4$ if and only if $6=0 ;-2=4 \omega$ if and only if $p-1=2 \omega$, which implies that $(p-1)^{3}=p-1=8$, and hence $p=9$ - both clear contradictions.

Up to conjugacy, there is only one proper parabolic subgroup of $G_{4}$, it is $\mathbb{Z}_{3}$. We may assume that $\mathbb{Z}_{3}=\left\langle s_{1}\right\rangle=\left\{1, s_{1}, t_{1}\right\}$. Repeating the above argument for $\mathbb{Z}_{3}$ and noting that $\operatorname{Irr} \mathbb{Z}_{3}=\left\{\left.T\right|_{\mathbb{Z}_{3}},\left.V_{1}\right|_{\mathbb{Z}_{3}},\left.V_{2}\right|_{\mathbb{Z}_{3}}\right\}$, one sees that the block partition of Irr $\mathbb{Z}_{3}$ will not consist of singletons precisely if equation (19) is satisfied for $\rho, \mu \in\left\{T, V_{1}, V_{2}\right\}$.

6.8. The Kac-Weisfeiler conjecture As in the previous section, we assume that $k$ is algebraically closed of characteristic $p>0$ and that $p$ is prime to the order of $W$, a pseudo-reflection group. Recall that the Kac-Weisfeiler conjecture, as stated in [35] and proved in [30], provides a lower bound on the power of $p$ dividing the dimension of a simple module over a restricted Lie algebra in terms of its support as a module for the $p$-centre. The following result considers, analogously, the powers of $p$ that divide the dimension of simple modules for the rational Cherednik algebra.

Proposition. The subset $\mathcal{C}^{\text {reg }}$ of $\mathcal{C}$ consisting of all parameters $\mathbf{c}$ such that the dimension of every simple $\mathrm{H}_{\mathbf{c}}(W)$-module is divisible by $p^{n}$ is open and dense.

Note that, since the P.I. degree of $\mathrm{H}_{\mathbf{c}}(W)$ is $p^{n}|W|, p^{n}$ is also the largest power of $p$ dividing the dimension of any simple $\mathrm{H}_{\mathbf{c}}(W)$-module. Also, one can show that $\mathcal{C}^{\text {reg }}$ is always a proper subset of $\mathcal{C}$.

Proof. Firstly, we show that the subset $\mathcal{C}^{\text {reg }}(W)$ of $\mathcal{C}$ consisting of all $\mathbf{c}$ such that the dimension of every simple $\overline{\mathrm{H}}_{\mathrm{c}}(W)$-module is divisible by $p^{n}$ is open and dense in $\mathcal{C}$. Consider the $k[\mathcal{C}]$-algebra $\overline{\mathrm{H}}_{\underline{\mathbf{c}}}(W)$. It is free as a $k[\mathcal{C}]$-module. Therefore, it is a continuous family of $\mathcal{L}$-algebras in the sense of [31, Definition 2.1] (for $\mathcal{L}$ trivial). Then [31, Lemma 2.2] says that, for each integer $d$, the set of points $\mathbf{c} \in \mathcal{C}$ such that $\overline{\mathrm{H}}_{\mathrm{c}}(W)$ contains a two-sided ideal of dimension $d$ is closed in $\mathcal{C}$. The annihilator of a 
simple $\overline{\mathrm{H}}_{\mathbf{c}}(W)$-module $M$ has codimension $\left(\operatorname{dim}_{k} M\right)^{2}$ (recall that $k$ is assumed to be algebraically closed). Therefore, the set of all points in $\mathcal{C}$ for which there exists an ideal in $\overline{\mathrm{H}}_{\mathbf{c}}(W)$ whose codimension belongs to $\left\{\left(p^{i} r\right)^{2}|0 \leq i<n, 1 \leq r \leq| W \mid\right\}$ is closed and its compliment is $\mathcal{C}^{\text {reg }}(W)$. We just need to show that this set is non-empty. Take any line $\ell$ in $\mathcal{C}$. Then Lemma 6.5 implies that $\ell \cap \mathcal{C}^{\text {reg }}(W)$ is dense in $\ell$. Note that this actually shows that there is no linear subspace of $\mathcal{C}$ contained in $\mathcal{C}-\mathcal{C}^{\text {reg }}(W)$.

Now we treat the general case. Let $W^{\prime}$ be a parabolic subgroup of $W$ and $\mathcal{C}_{W^{\prime}}$ the space of parameters for $\mathrm{H}_{\mathbf{c}}\left(W^{\prime}\right)$. Restriction of parameters defines a linear map $\rho: \mathcal{C} \rightarrow \mathcal{C}_{W^{\prime}}$, its image is a non-zero linear subspace of $\mathcal{C}_{W^{\prime}}$. We have shown that the set of points in $\mathcal{C}_{W^{\prime}}$ for which all simple $\overline{\mathrm{H}}_{\mathbf{c}}\left(W^{\prime}\right)$-module has 'maximum $p$-dimension' is open and dense. If $\mathcal{C}^{\text {reg }}\left(W^{\prime}\right)$ denotes the pre-image of this set under $\rho$, then it is open and dense in $\mathcal{C}$. Then it follows from Propositions 5.3 and 5.5 together with Corollary 5.5 that

$$
\mathcal{C}^{\text {reg }}=\bigcap_{W^{\prime}} \mathcal{C}^{\text {reg }}\left(W^{\prime}\right)
$$

where the intersection is over all conjugacy classes of parabolic subgroups of $W$.

REMARK. We remark that our reduction method, combining Propositions 5.3 and 5.5 together with Corollary 5.5, also gives a different proof of the result [34, Corollary 4.2] (which is closer in spirit to the original Kac-Weisfeiler conjecture): If $M$ is a simple $\mathrm{H}_{\mathbf{c}}(W)$ and $b \in V$ such that the support of $M$ equals the image of $b$ in $V / W$ then $p^{\left|W / W_{b}\right|}$ divides $\operatorname{dim}_{k} M$.

EXAMPLE. When $W=S_{n+1}, \mathcal{C}^{\text {reg }}=k-\mathbb{F}_{p}^{\times}$. One can show this as follows: By remark 5.5, the algebra $\mathrm{H}_{\mathbf{c}}\left(S_{n+1}\right)$ is Azumaya for all $\mathbf{c} \in k-\mathbb{F}_{p}$ and hence $k-\mathbb{F}_{p} \subseteq \mathcal{C}^{\text {reg }}$. On the other hand, direct calculations show that $\mathcal{C}-\mathcal{C}^{\text {reg }}\left(S_{2}\right)=\mathbb{F}_{p}^{\times}$. Therefore, Propositions 5.3 implies that $\mathbb{F}_{p}^{\times} \subset \mathcal{C}-\mathcal{C}^{\text {reg }}$. Finally, when $\mathbf{c}=0, \mathrm{H}_{\mathbf{c}}\left(S_{n+1}\right)=\mathcal{D}(V) \rtimes S_{n+1}$ and, even though this is not an Azumaya algebra, it follows from Lemma 6.2 that $p^{n}$ does divide $\operatorname{dim}_{k} L$ for all simple $\mathcal{D}(V) \rtimes S_{n+1}$-modules $L$.

ACKNOWLEDGEMENTS. The first author was supported by the EPSRC grant EPH028153. The second author was supported by the SFB/TR 45 'Periods, Moduli Spaces and Arithmetic of Algebraic Varieties' of the DFG (German Research Foundation). The authors are grateful for the hospitality of the Max Planck Institute for Mathematics in Bonn. The authors would like to thank Ulrich Thiel for suggesting that the Euler element should distinguish the blocks for $G_{4}$ at generic parameters.

\section{REFERENCES}

1. M. Balagovic and $\mathrm{H}$. Chen, Category $\mathcal{O}$ for rational Cherednik algebras $H_{t, c}\left(G L_{2}\left(F_{p}\right), h\right)$ in characteristic p, J. Pure Appl. Algebra 217(9) (2013), 1683-1699.

2. M. Balagovic and H. Chen, Representations of rational Cherednik algebras in positive characteristic, J. Pure Appl. Algebra 217(4) (2013), 716-740. $315-326$.

3. G. Bellamy, On singular Calogero-Moser spaces, Bull. London Math. Soc. 41(2) (2009), $47-77$.

4. G. Bellamy, The Calogero-Moser partition for $G(m, d, n)$, Nagoya Math. J. 207 (2012),

5. R. Bezrukavnikov and P. Etingof, Parabolic induction and restriction functors for rational Cherednik algebras, Selecta Math. (NS) 14(3-4) (2009), 397-425. 
6. R. Bezrukavnikov, M. Finkelberg and V. Ginzburg, Cherednik algebras and Hilbert schemes in characteristic $p$, Represent. Theory 10 (2006), 254-298, . With an appendix by Pavel Etingof.

7. K. A. Brown and K. Changtong, Symplectic reflection algebras in positive characteristic, Proc. Edinb. Math. Soc., 53(1) (2010), 61-81.

8. K. A. Brown and I. G. Gordon, The ramification of centres: Lie algebras in positive characteristic and quantised enveloping algebras, Math. Z. 238(4) (2001), 733-779.

9. K. A. Brown, I. G. Gordon and C. H. Stroppel, Cherednik, Hecke and quantum algebras as free Frobenius and Calabi-Yau extensions, J. Algebra 319(3) (2008), 1007-1034.

10. C. W. Curtis and I. Reiner, Methods of representation theory, Vol. I; with applications to finite groups and orders, Pure and Applied Mathematics series (John Wiley, New York, NY, 1981).

11. C. Dunkl and S. Griffeth, Generalized Jack polynomials and the representation theory of rational Cherednik algebras, Selecta Math. (NS) 16(4) (2010), 791-818.

12. C. F. Dunkl and E. M. Opdam, Dunkl operators for complex reflection groups, Proc. London Math. Soc., 86(1) (2003), 70-108.

13. D. Eisenbud, Commutative algebra, Graduate Texts in Mathematics, vol. 150. (SpringerVerlag, New York, 1995). With a view toward algebraic geometry.

14. P. Etingof and V. Ginzburg, Symplectic reflection algebras, Calogero--Moser space, and deformed Harish-Chandra homomorphism, Invent. Math. 147(2) (2002), 243-348.

15. M. Geck and G. Pfeiffer, Characters of finite coxeter groups and Iwahori-Hecke algebras, London Mathematical Society Monographs New Series, vol. 21. (Clarendon Press/Oxford University Press, New York, NY, 2000).

16. V. Ginzburg and D. Kaledin, Poisson deformations of symplectic quotient singularities, Adv. Math. 186(1) (2004), 1-57.

17. I. G. Gordon, Baby Verma modules for rational Cherednik algebras, Bull. London Math. Soc. 35(3) (2003), 321-336.

18. I. G. Gordon, Quiver varieties, category $\mathcal{O}$ for rational Cherednik algebras and Hecke algebras, International Mathematics Research Papers (IMRP), vol. 2008(3) (2008), Art. ID: rpn006, 69; doi:10.1093/imrp/rpn006.

19. S. Griffeth, Orthogonal functions generalizing Jack polynomials, Trans AMS 362 (2010), 6131-6157.

20. S. Griffeth, Towards a combinatorial representation theory for the rational Cherednik algebra of type G(r,p,n), Proc. Edinb. Math. Soc., 53(2) (2010), 419-445.

21. R. R. Holmes and D. K. Nakano, Brauer-type reciprocity for a class of graded associative algebras, J. Algebra 144(1) (1991), 117-126.

22. G. James and A. Kerber, The representation theory of the symmetric group, Encyclopedia of Mathematics and its Applications, vol. 16 (Addison-Wesley, Reading, MA, 1981) (with a foreword by P. M. Cohn, and an introduction by Gilbert de B. Robinson).

23. N. M. Katz, Nilpotent connections and the monodromy theorem: Applications of a result of Turrittin, Inst. Hautes Études Sci. Publ. Math. (39) (1970), 175-232.

24. G. Kemper and G. Malle, The finite irreducible linear groups with polynomial ring of invariants, Transform. Groups, 2(1) (1997), 57-89.

25. G. I. Lehrer and D. E. Taylor, Unitary reflection groups, Australian Mathematical Society Lecture Series, vol. 20 (Cambridge University Press, Cambridge, UK, 2009).

26. M. Martino, Stratifications of Marsden-Weinstein reductions for representations of quivers and deformations of symplectic quotient singularities, Math. Z. 258(1) (2008), 1-28.

27. M. Martino, Blocks of restricted rational Cherednik algebras for $G(m, d, n)$ (to appear) J. Algebra.

28. M. Martino, The Calogero-Moser partition and Rouquier families for complex reflection groups, J. Algebra 323 (2010), 193-205.

29. J. C. McConnell and J. C. Robson, Noncommutative noetherian rings, Graduate Studies in Mathematics, vol. 30 (American Mathematical Society, Providence, RI, 2001, revised edition) (with the cooperation of L. W. Small).

30. A. Premet, Irreducible representations of Lie algebras of reductive groups and the Kac-Weisfeiler conjecture, Invent. Math. 121(1) (1995), 79-117.

31. A. Premet and S. Skryabin, Representations of restricted Lie algebras and families of associative $\mathcal{L}$-algebras, J. Reine Angew. Math. 507 (1999), 189-218. 
32. I. A. Pushkarev, On the theory of representations of the wreath products of finite groups and symmetric groups, Zap. Nauchn. Sem. S.-Peterburg. Otdel. Mat. Inst. Steklov. (POMI) 240 (1997), 229-244; Teor. Predst. Din. Sist. Komb. i Algoritm. Metody. 2 (1997), 294-295.

33. L. Smith, Polynomial invariants of finite groups, Research Notes in Mathematics, vol. 6 (A K Peters, Wellesley, MA, 1995).

34. A. Tikaradze, An analogue of the Kac-Weisfeiler conjecture, J. Algebra 383 (2013), $168-177$.

35. B. Ju. Veřsfeĭler and V. G. Kac, The irreducible representations of Lie p-algebras, Funkcional. Anal. i Priložen. 5(2) (1971), 28-36. 